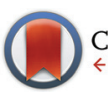

CrossMark click for updates

Cite this: Org. Biomol. Chem., 2015, 13, 4539

Received 5th February 2015,

Accepted 3rd March 2015

DOI: $10.1039 / c 5 o b 00251 f$

www.rsc.org/obc

\section{Chirality extension of an oxazine building block en route to total syntheses of (+)-hyacinthacine $A_{2}$ and sphingofungin $\mathrm{B} \dagger$}

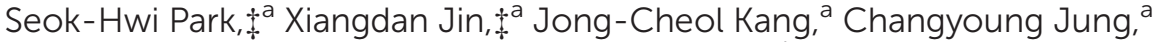 \\ Seong-Soo Kim, ${ }^{a}$ Sung-Soo Kim, ${ }^{a}$ Kee-Young Lee ${ }^{b}$ and Won-Hun Ham*a
}

\begin{abstract}
Concise and stereocontrolled syntheses of (+)-hyacinthacine $A_{2}$ and sphingofungin $B$ were achieved via a diastereomerically enriched oxazine intermediate. The key strategies include palladium(0)-catalyzed intramolecular oxazine formation and diastereoselective nucleophilic addition to an aldehyde. (+)-Hyacinthacine $A_{2}$ was synthesized in 13 steps and $10.2 \%$ overall yield and the synthesis of sphingofungin $B$ proceeded in a linear sequence over 15 steps and 6.9\% overall yield from $(R)$-methyl 2-benzamido3-((tert-butyldimethylsilyl)oxy)propanoate.
\end{abstract}

\section{Introduction}

As part of an ongoing research program aimed at developing total syntheses of biologically active compounds, we recently described a facile strategy towards the preparation of syn,syn-, syn,anti-, ${ }^{1}$ and anti,syn-oxazines ${ }^{2}$ via a stereoselective palladium(0)-catalyzed reaction (Scheme 1). The diastereoselectivity
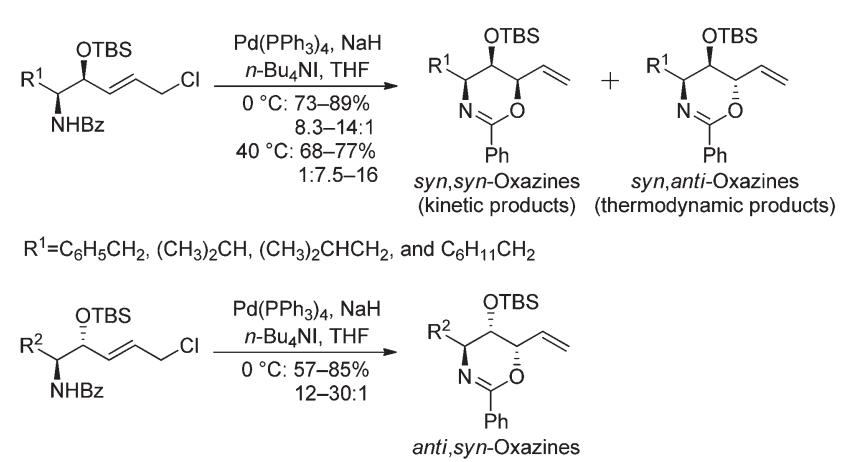

$\mathrm{R}^{2}=\mathrm{C}_{6} \mathrm{H}_{5}, \mathrm{C}_{6} \mathrm{H}_{5} \mathrm{CH}_{2},\left(\mathrm{CH}_{3}\right)_{2} \mathrm{CH},\left(\mathrm{CH}_{3}\right)_{2} \mathrm{CHCH}_{2}$, and $\mathrm{TBSOCH}_{2}$

Scheme 1 Oxazine library generated via a stereoselective palladium(0)catalyzed reaction.

\footnotetext{
${ }^{a}$ School of Pharmacy, Sungkyunkwan University, Suwon 440-746, Republic of Korea. E-mail:whham@skku.edu; Fax: +82 31292 8800; Tel: +82 312907706

${ }^{b}$ Yonsung Fine Chemicals Co., Ltd, Hwaseong 445-944, Republic of Korea. E-mail: uncleduly@skku.edu; Fax: +82 31351 6624; Tel: +82 313516622 $\dagger$ Electronic supplementary information (ESI) available: Copies of ${ }^{1} \mathrm{H}$ and ${ }^{13} \mathrm{C}$ NMR spectra of $\mathbf{1}, 2$, and all new compounds; and NOESY spectra of trans-6 and cis-6. See DOI: 10.1039/c5ob00251f

$\$$ These authors contributed equally.
}

of syn,syn- and syn,anti- cyclizations can be critically dependent upon whether the reaction temperature results in kinetic or thermodynamic control of the products. Meanwhile, the diastereoselectivity of anti,syn-oxazine ring formation is predominantly controlled by the bulky OTBS group. Based on the oxazine library, piperidine alkaloid $\mathrm{D}$-fagomine ${ }^{2 d}$ pyrrolidine alkaloid DAB-1, ${ }^{2 d}$ indolizidine alkaloid (-)-lentiginosine, ${ }^{2 b}$ phytosphingosines ${ }^{1 a, 2 c}$ and other natural products bearing three contiguous chiral centers were successfully synthesized.

Polyhydroxylated alkaloids (or aminopolyols) isolated from plants and microorganisms should be thoroughly investigated because they can act as glycosidase inhibitors by mimicking natural monosaccharide substrates, which may allow for the development of new antiviral, antidiabetic, and anticancer agents. ${ }^{3}$ More than two hundred of these naturally-occurring and water-soluble compounds have been isolated and classified structurally as piperidines, pyrrolidines, pyrrolizidines, indolizidines, and nortropanes. (+)-Hyacinthacine $\mathrm{A}_{2}$ (1), which is a representative pyrrolizidine alkaloid, was first isolated from Muscari armeniacum bulbs and is a good inhibitor of both amyloglucosidase and lactase (Fig. 1). ${ }^{4}$

Its promising biological properties and the existence of four contiguous stereogenic centers in its structure have prompted the development of numerous synthetic approaches. ${ }^{5,6}$ For example, in 2011, Fox et al. described the concise synthesis of (+)-hyacinthacine $\mathrm{A}_{2}$, which relied on a novel transannular hydroamination of 5-aza-cyclooctene. ${ }^{5 a}$ Zheng and Huang's approach for the synthesis of $\mathbf{1}$ was $\mathrm{SmI}_{2}$-mediated radical coupling reaction of an activated amide from $(R, R)$-tartarimide. ${ }^{5 b}$ Bonaccini et al. reported a stereocontrolled cyclic nitrone cycloaddition for the synthesis of $1 .^{5 c}$ Another previous result by Goti and Merino started from a nitrone, which is 


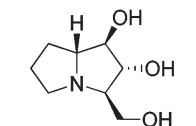

(+)-Hyacinthacine $\mathrm{A}_{2} 1$<smiles>OCC1NCCC(O)C1O</smiles>

D-Fagomine

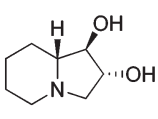

$(-)$-Lentiginosine

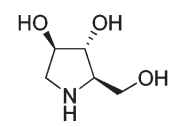

DAB-1
Fig. 1 The structures of several polyhydroxylated alkaloids.

readily prepared from D-arabinose ${ }^{5 e}$ Marco et al. demonstrated a stereoselective synthesis of $\mathbf{1}$ via a double cyclization with the one-pot formation of two C-N bonds..$^{5 f}$

Sphingofungins isolated from fungi are also of significant interest owing to their biological activities as immunosuppressants and potential antifungal agents via the inhibition of serine palmitoyl-CoA transferase (SPT). ${ }^{7}$ In addition to sphingofungins, congeners such as myriocin, sulfamisterin, and mycestericins exhibit similar functions. ${ }^{7}$ Sphingofungin B (2), having a polar polyhydroxy amino acid head group and a long lipid chain containing an $(R)$-hydroxy group at $\mathrm{C}-14$, was isolated from the fermentation broth of Aspergillus fumigatus and its structure was elucidated by Merck group in 1992 (Fig. 2). ${ }^{8}$

The promising biological activities and more than three contiguous chiral centers present in these compounds also have prompted the development of numerous synthetic approaches. ${ }^{9-15}$ For example, the first total synthesis of sphingofungin B was successfully achieved by Kobayashi et al. in 1996 making use of catalytic asymmetric aldol reactions. ${ }^{9}$ Meanwhile, the first total synthesis of sphingofungin D was

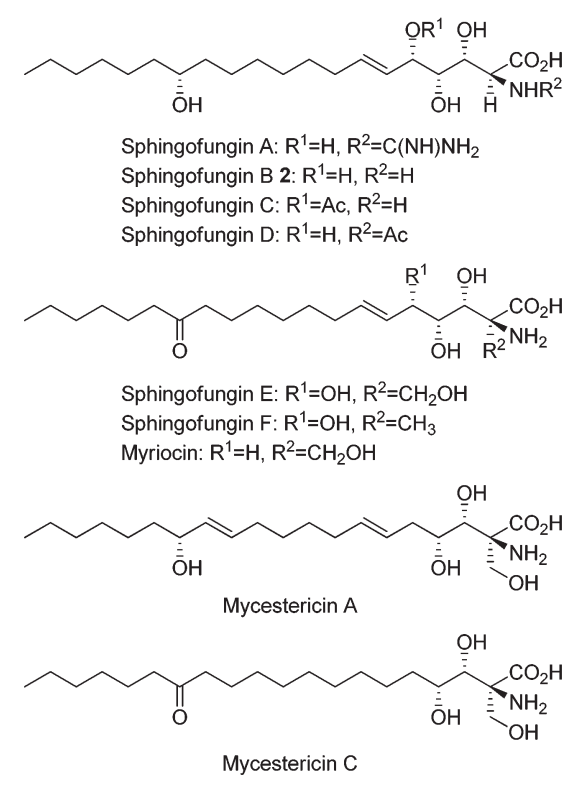

Fig. 2 The structures of several sphingolipids. reported by Mori et al. in 1994 using a polar building block derived from $N$-acetyl-D-mannoside. ${ }^{10 a, c}$ Shortly thereafter, Chida et al. accomplished the synthesis of sphingofungin D starting from myo-inositol. ${ }^{10 b}$

Herein we report the highly stereocontrolled total syntheses of (+)-hyacinthacine $\mathrm{A}_{2}$ and sphingofungin $\mathrm{B}$ via straightforward procedures that rely on a chiral anti,syn-oxazine building block.

\section{Results and discussion}

\section{Chirality extension of a chiral anti,syn-oxazine}

The first step in the syntheses, the preparation of the known chiral 1,3-oxazine 4 beginning with $(R)$-methyl 2-benzamido-3((tert-butyldimethylsilyl)oxy)propanoate 3, proceeded in $56.8 \%$ yield after five steps (Scheme 2). ${ }^{2 b, d}$

We envisioned that a stereocontrolled Grignard reaction or Grignard-like nucleophilic addition ${ }^{16}$ to the oxazine-derived aldehyde could generate the desired fourth contiguous stereocenter. In most cases, the chelation-control product of a Grignard reaction is produced via the chelation of organomagnesium nucleophiles rather than bisligation of Lewis acids; this is why ethers, which are the universal solvents of Grignard reactions, coordinate to Lewis acids to form octahedral complexes. ${ }^{16 e, 17}$ Therefore, it is uncertain whether Lewis acid additives such as $\mathrm{ZnCl}_{2},{ }^{18,19} \mathrm{ZnBr}_{2},{ }^{18-21} \mathrm{ZnI}_{2}{ }^{22,23}$

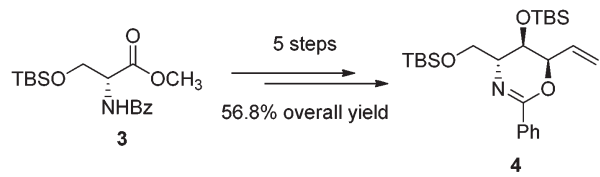

Scheme 2 Synthesis of anti,syn-oxazine 4.

Table 1 Grignard reaction with the prepared aldehyde

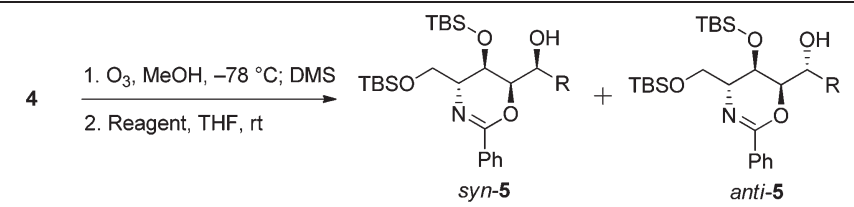

$R=\operatorname{vinyl}(5 a)$, methyl (5b), ethyl (5c), allyl (5d), homoallyl (5e), and phenyl (5f)

\begin{tabular}{|c|c|c|c|c|c|}
\hline Entry & Reagent & $\begin{array}{l}\text { Time } \\
\text { (h) }\end{array}$ & Product & $\begin{array}{l}\text { Ratio }^{a} \\
(\text { syn/anti) }\end{array}$ & $\begin{array}{l}\text { Yield }^{b} \\
(\%)\end{array}$ \\
\hline 1 & $\mathrm{CH}_{2} \mathrm{CHMgBr}$ & 0.5 & $5 \mathbf{a}$ & $1.3: 1$ & 78 \\
\hline 2 & $\mathrm{MeMgBr}$ & 0.5 & $5 \mathbf{b}$ & $1: 1.5$ & 71 \\
\hline 3 & EtMgBr & 0.5 & $5 c$ & $1.5: 1$ & 70 \\
\hline 4 & $\mathrm{CH}_{2} \mathrm{CHCH}_{2} \mathrm{MgBr}$ & 0.5 & $5 d$ & $2.0: 1$ & 70 \\
\hline 5 & $\mathrm{CH}_{2} \mathrm{CH}\left(\mathrm{CH}_{2}\right)_{2} \mathrm{MgBr}$ & 0.5 & $5 e$ & $1.5: 1$ & 70 \\
\hline 6 & PhMgBr & 0.5 & $5 f$ & $4: 1$ & 70 \\
\hline
\end{tabular}

${ }^{a}$ Ratios were determined by ${ }^{1} \mathrm{H}$ NMR peak intensities. ${ }^{b}$ Yields refer to isolated yields over the two steps. 
$\mathrm{MgBr}_{2} \cdot \mathrm{OEt}_{2},{ }^{20,23,24} \mathrm{CdCl}_{2},{ }^{18}$ and $\mathrm{Ti}(\mathrm{OiPr})_{4}{ }^{20}$ act as bidentate ligands, although stereoselective addition should be possible via a chelated transition state. ${ }^{25}$ Hence, $\mathrm{ZnCl}_{2}$ was tested in the following reaction to optimize the diastereomeric enrichment.

The results of the introduction of Grignard reagents are shown in Table 1. Unfortunately, in the absence of additives, organomagnesiums cannot chelate. Meanwhile, Table 2 summarizes the results of reactions in which $\mathrm{ZnCl}_{2}$ was added to the reaction mixture; in each case, 1.1 equivalents of the Lewis acid were added dropwise followed by the addition of 3.0 equivalents of each Grignard reagent. Surprisingly, reactions favored the syn-product, indicating the occurrence of $\alpha$-chelation with the oxygen inside the 1,3-oxazine moiety.

Table $2 \mathrm{ZnCl}_{2}$-mediated Grignard reaction with the prepared aldehyde

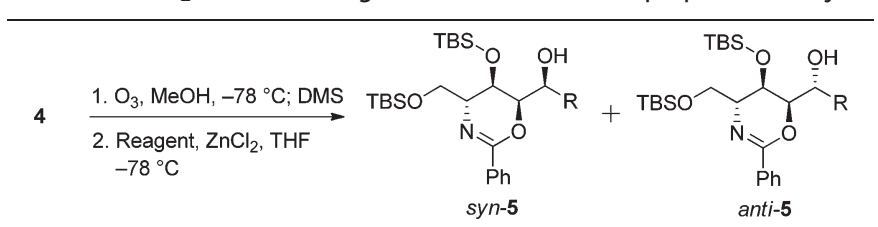

$\mathrm{R}=\operatorname{vinyl}(\mathbf{5 a})$, methyl $(5 \mathbf{b})$, ethyl (5c), allyl (5d), homoallyl (5e), and phenyl (5f)

\begin{tabular}{llllll}
\hline Entry & Reagent & $\begin{array}{l}\text { Time } \\
(\mathrm{h})\end{array}$ & Product & $\begin{array}{l}\text { Ratio }^{a} \\
(\text { syn/anti })\end{array}$ & $\begin{array}{l}\text { Yield }^{b} \\
(\%)\end{array}$ \\
\hline 1 & $\mathrm{CH}_{2} \mathrm{CHMgBr}$ & 1 & $\mathbf{5 a}$ & $10: 1$ & 62 \\
2 & $\mathrm{MeMgBr}_{3}$ & 1 & $\mathbf{5 b}$ & $2.2: 1$ & 59 \\
3 & EtMgBr & 1 & $\mathbf{5 c}$ & $14: 1$ & 60 \\
4 & $\mathrm{CH}_{2} \mathrm{CHCH}{ }_{2} \mathrm{MgBr}$ & 1 & $\mathbf{5 d}$ & $>20: 1$ & 60 \\
5 & $\mathrm{CH}_{2} \mathrm{CH}\left(\mathrm{CH}_{2}\right)_{2} \mathrm{MgBr}$ & 1 & $\mathbf{5 e}$ & $>20: 1$ & 62 \\
6 & $\mathrm{PhMgBr}$ & 1 & $\mathbf{5 f}$ & $8: 1$ & 65
\end{tabular}

${ }^{a}$ Ratios were determined by ${ }^{1} \mathrm{H}$ NMR peak intensities. ${ }^{b}$ Yields refer to isolated yields over the two steps.
Further experiments for using other organometallic compounds were conducted (Table 3 ). Replacing magnesium with lithium, which is less prone to chelation, shifted the product composition slightly to favor the Felkin-Anh variant (entry 1). Vinylation using vinylzinc chloride and divinylzinc, which were prepared in situ from zinc chloride and vinylmagnesium bromide, ${ }^{26}$ generated the syn-alcohol exclusively (entries 2-4). Unfortunately, these conditions have limited applications; reactions using $\mathrm{Me}_{2} \mathrm{Zn}, \mathrm{Et}_{2} \mathrm{Zn}$, and other reagents proceeded sluggishly (entries 5-8), while that involving $\mathrm{Ph}_{2} \mathrm{Zn}$ proceeded much more quickly (entry 9).

To determine the relative stereochemistry of syn-5a, diastereoisomer anti-5a was prepared (Table 1, entry 1). Oxazine rings of syn-5a and anti-5a were cleaved by hydrogenolysis. Two secondary alcohols gave the corresponding acetals, respectively. Unfortunately, the coupling constants between $\mathrm{H}_{4}$ and $\mathrm{H}_{5}$ of trans-6 and cis-6 were not in good agreement with theoretical values $\left[J_{4,5}\left(\right.\right.$ trans-6) $=8.0 \mathrm{~Hz}$ and $J_{4,5}($ cis-6) $=5.4 \mathrm{~Hz}]$. Therefore, NOESY spectra of trans-6 and cis-6, representing the correlations between $\mathrm{H}_{4}$ or $\mathrm{H}_{5}$ and acetal methyl protons, confirm the identification of the diastereoisomers (Scheme 3).

\section{Total synthesis of (+)-hyacinthacine $\mathbf{A}_{2}$}

Our retrosynthesis (Scheme 4) suggests that the bicyclic structure of 1 could be synthesized from tertiary amine 7 , which could in turn be prepared from secondary mesylate 8 via intramolecular $\mathrm{S}_{\mathrm{N}} 2$ substitution. Conveniently, mesylate 8 could be obtained from the pentenyl alcohol syn-5e. The synthesis of syn-5e could be accomplished by nucleophilic addition to the corresponding aldehyde, which could be derived from conversion of the exomethylene moiety of anti,syn-oxazine 4 .

Pentenyl alcohol syn-5e was consecutively treated with $\mathrm{MsCl}$ and benzyl chloroformate under biphasic conditions to afford carbamate 8 in $69 \%$ yield over 2 steps. The room temperature exposure of $\mathbf{8}$ to sodium hydride led to the formation of ter-

Table 3 Organometallic nucleophilic addition with the prepared aldehyde

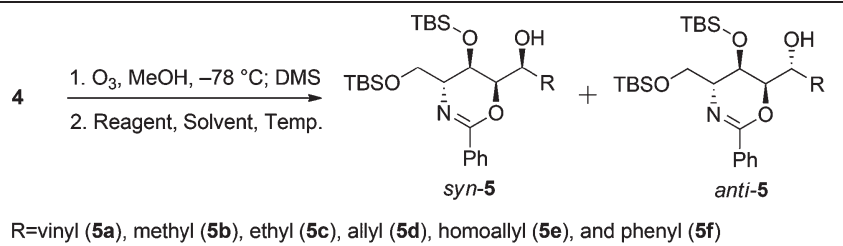

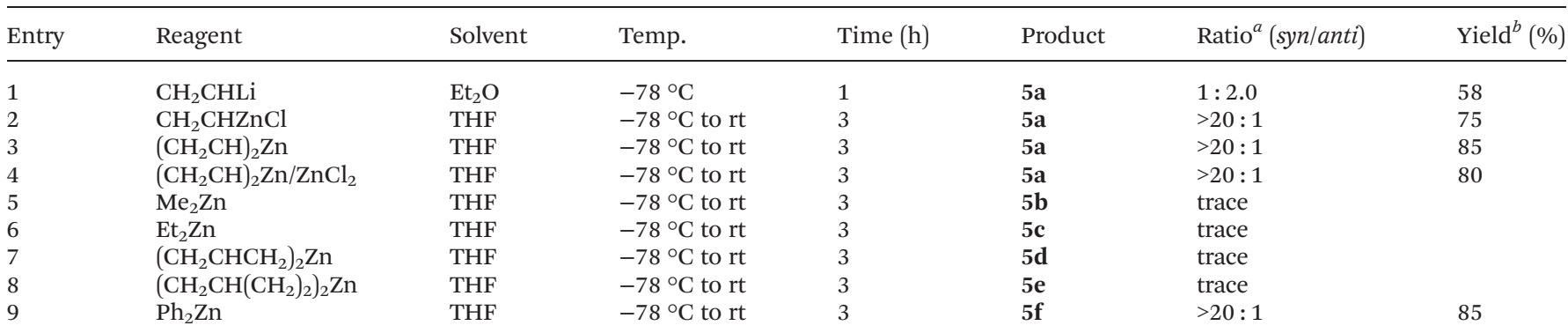

${ }^{a}$ Ratios were determined by ${ }^{1} \mathrm{H}$ NMR peak intensities. ${ }^{b}$ Yields refer to isolated yields over the two steps. 

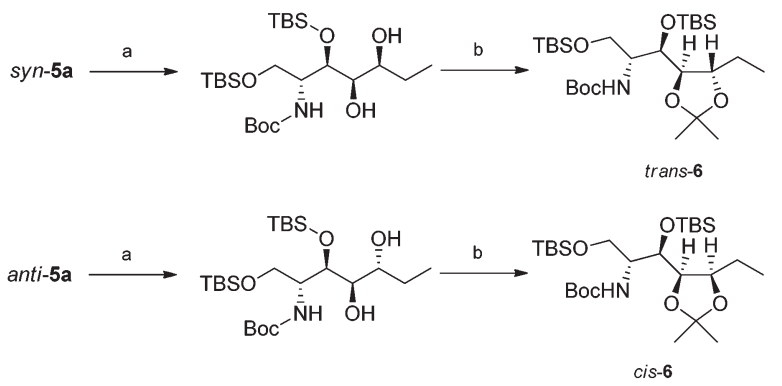

(a) $\mathrm{Pd}(\mathrm{OH})_{2} / \mathrm{C}, \mathrm{H}_{2}, \mathrm{Boc}_{2} \mathrm{O}$, rt; (b) $\left(\mathrm{CH}_{3}\right)_{2} \mathrm{C}\left(\mathrm{OCH}_{3}\right)_{2}$, rt, both $90 \%$ (for 2 steps)

Scheme 3 Determination of the relative stereochemistry of trans- 6 and cis-6.
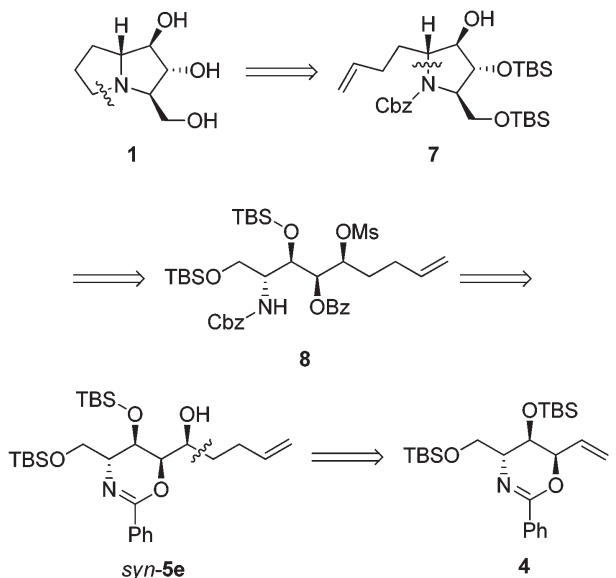

Scheme 4 Retrosynthesis of 1.

tiary amine 7 (mixture of rotamers) in $83 \%$ yield via intramolecular cyclization as well as $O$-benzoyl hydrolysis. Next, ozonolysis and hydrogenolysis of 7 gave protected 9 in $72 \%$ yield over 2 steps. Finally, removal of TBS-protecting groups by treatment of 9 with concentrated acid yielded the $\mathbf{1} \cdot \mathrm{HCl}$ salt, which was neutralized with an ion-exchange resin to give 1 in $70 \%$ yield (Scheme 5). Our $[\alpha]_{\mathrm{D}}+15.3\left(c 0.1, \mathrm{H}_{2} \mathrm{O}\right)$ compared to the reported $[\alpha]_{\mathrm{D}}+20.1\left(c 0.44, \mathrm{H}_{2} \mathrm{O}\right),{ }^{4}[\alpha]_{\mathrm{D}}+12\left(c 0.40, \mathrm{H}_{2} \mathrm{O}\right),{ }^{5 a}[\alpha]_{\mathrm{D}}$ $+10.6\left(c 1.64, \mathrm{H}_{2} \mathrm{O}\right),{ }^{5 b}$ and $[\alpha]_{\mathrm{D}}+10.5\left(c 0.6, \mathrm{H}_{2} \mathrm{O}\right)^{5 i}$ confirms the identity of the absolute configuration. We could also confirm the relative stereochemistry of chromatographically separable syn-5e after comparing spectroscopic data of $\mathbf{1}$ with those of 7a-epimer, 7-deoxyalexine. ${ }^{27}$

\section{Total synthesis of sphingofungin B}

Our retrosynthesis (Scheme 6) suggested that 2 could be generated from allylic alcohol syn-5a and lipid chain $\mathbf{1 0}$ by an olefin cross-metathesis reaction. Chiral alcohol 10 could be obtained from $(R)$-epoxyoctane 11 and 6-heptenylmagnesium bromide 12 .

Subsequent acetylation of the hydroxyl group of syn-5a yielded secondary allylic acetate 13 in 94\% yield. Meanwhile, the nucleophilic addition reaction between $(R)$-epoxyoctane $\mathbf{1 1}$
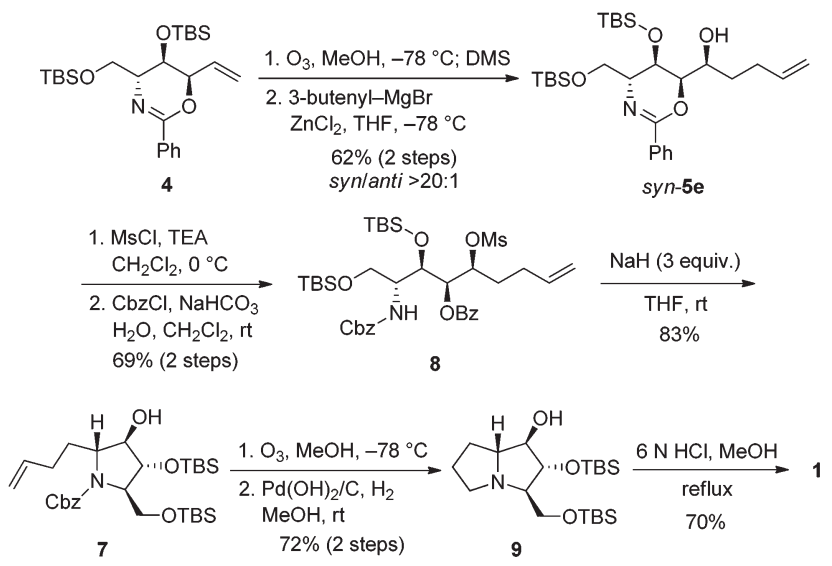

Scheme 5 Synthesis of 1.

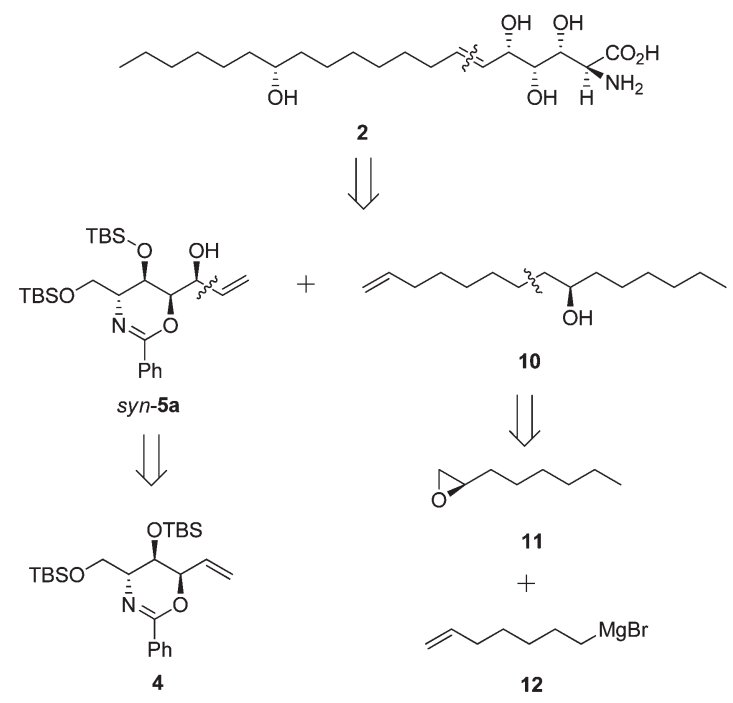

Scheme 6 Retrosynthesis of 2.

and 6-heptenylmagnesium bromide 12, which are both commercially available, and the subsequent acetylation provided the lipid chain subunit 14 in $85 \%$ yield over two steps (Scheme 7).

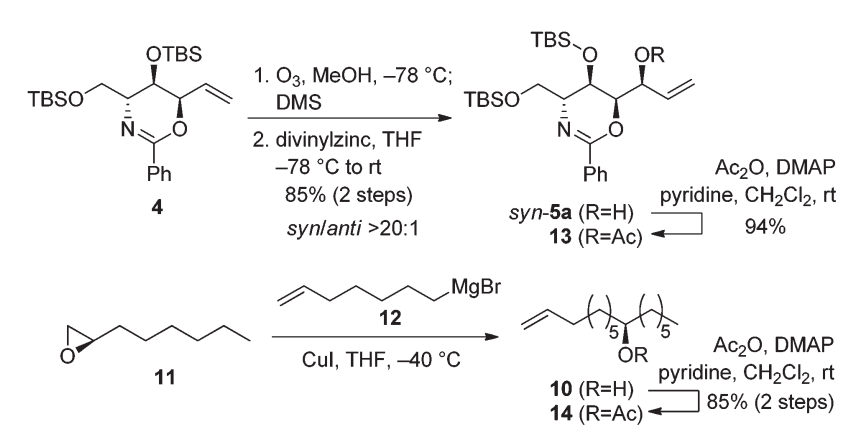

Scheme 7 Syntheses of subunits 13 and 14. 

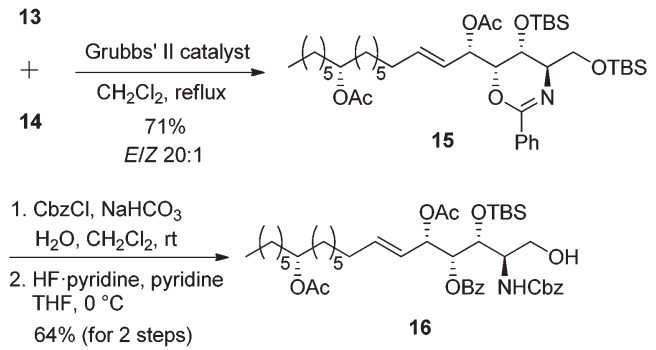

$64 \%$ (for 2 steps)

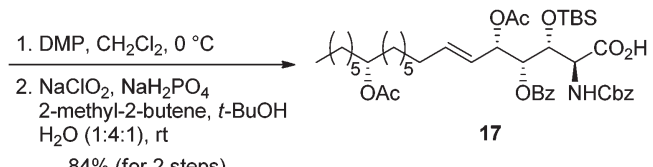

$84 \%$ (for 2 steps)

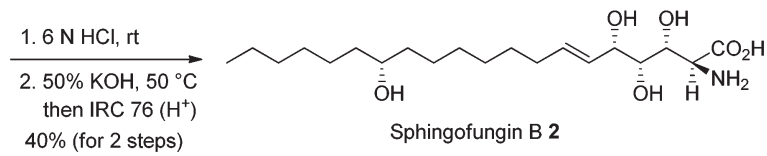

Scheme 8 Synthesis of 2

Intermolecular olefin cross-metathesis between 13 and $\mathbf{1 4}$ resulted in compound 15 in 71\% yield ( $E / Z>20: 1$ ratio). 1,3Oxazine 15 was treated with benzyl chloroformate under biphasic conditions followed by primary OTBS deprotection affording carbamate 16 in 64\% yield over two steps. Primary alcohol 16 was oxidized to carboxylic acid 17 in 84\% yield over two steps. Finally, acid- and base-promoted hydrolysis cleaved all protecting groups, and subsequent neutralization afforded sphingofungin B 2 in 40\% yield over two steps (Scheme 8). The synthetic compound was spectroscopically in good agreement with the reported sphingofungin B. ${ }^{9}$

\section{Conclusions}

We described new procedures for stereoselective nucleophilic addition to anti,syn-oxazine. The diastereoselectivity of the Grignard reaction was predominantly controlled by zinc chloride. Furthermore, the reactions that used divinylzinc and diphenylzinc also favored the syn-adducts. We took advantage of these reactions and various other available transformations to synthesize (+)-hyacinthacine $\mathrm{A}_{2}$ (1) and sphingofungin $\mathrm{B}$ (2) from a common source via chiral alcohol syn-5e and syn-5a. In addition to the diastereoselective reactions, these syntheses rely on a palladium(0)-catalyzed intramolecular oxazine formation reaction. Starting from readily available 3, 1 was obtained in 13 steps and $10.2 \%$ overall yield, whereas starting from oxazine chiral building block 4, 1 was afforded in 8 steps and $17.9 \%$ overall yield. Meanwhile, the synthesis of 2 proceeded in a linear sequence beginning from 3 over 15 steps and $6.9 \%$ overall yield and from the chiral oxazine 4 over 10 steps and $12.2 \%$ overall yield. The main advantage of this strategy is its high versatility, which allows the synthesis of not only 1 and 2, but also a range of structural analogs. Using this protocol, we are in the process of synthesizing castanosper- mines, australine, broussonetines, and other natural products; the results will be reported in due course.

\section{Experimental}

\section{General methods}

Flash chromatography was executed using a Merck Kieselgel 60 (230-400 mesh) stationary phase and mixtures of ethyl acetate and hexanes as the eluents. Ethyl acetate and hexanes were dried and purified by distillation prior to use. Tetrahydrofuran and diethyl ether were distilled over sodium and benzophenone (indicator). Dichloromethane was mixed with concentrated sulfuric acid (Caution), dried over potassium carbonate, and distilled. Commercially available compounds were used without further purification. ${ }^{1} \mathrm{H}$ and ${ }^{13} \mathrm{C}$ NMR spectra were recorded at the Center for Cooperative Research Facility at Sungkyunkwan University on FT-NMR 500 and $700 \mathrm{MHz}$ spectrometers. Chemical shifts are reported as $\delta$ values in ppm relative to the $\mathrm{CHCl}_{3}$ residual peak (7.26 ppm in $\mathrm{CDCl}_{3}$ ). IR spectra were recorded on a Bruker FT-IR spectrometer. Optical rotation was measured on a Jasco Dip 1020 digital polarimeter. Mass spectral data were recorded at the Korea Basic Science Institute (Daegu) on a Jeol JMS 700 high resolution mass spectrometer.

$(S)-1-((4 R, 5 R, 6 R)-5-(($ tert-Butyldimethylsilyl)oxy)-4-((tertbutyldimethylsilyl)oxy)methyl)-2-phenyl-5,6-dihydro-4H-1,3oxazin-6-yl)prop-2-en-1-ol (syn-5a). Oxazine $4 \quad$ (50 mg, $0.108 \mathrm{mmol})$ was dissolved in dry methanol $(2.16 \mathrm{~mL})$ and cooled to $-78^{\circ} \mathrm{C}$. Ozone was then passed through the solution until the reaction was complete. The reaction mixture was quenched with methyl sulfide $(0.08 \mathrm{~mL})$ and allowed to warm to rt. The solvent was evaporated under reduced pressure, and the crude aldehyde was immediately employed in the next step without further purification. Vinylmagnesium bromide $(1.0 \mathrm{M}$ solution in diethyl ether, $0.32 \mathrm{~mL}, 0.324 \mathrm{mmol}$ ) was added to a solution of zinc chloride $(1.0 \mathrm{M}$ solution in diethyl ether, $0.32 \mathrm{~mL}, 0.324 \mathrm{mmol})$ in THF $(0.76 \mathrm{~mL})$ at rt and stirred for $0.5 \mathrm{~h}$. A solution of the crude aldehyde in THF $(0.32 \mathrm{~mL})$ was added to this white suspension at $-78{ }^{\circ} \mathrm{C}$ and allowed to warm to rt. After $3 \mathrm{~h}$, the reaction was quenched with saturated $\mathrm{NH}_{4} \mathrm{Cl}$. The organic layer was separated and the aqueous layer was extracted with ethyl acetate. The combined organic layer was washed with saturated $\mathrm{NaHCO}_{3}$ and brine, dried with $\mathrm{MgSO}_{4}$, and filtered. The filtrate was concentrated in vacuo. Purification using silica gel column chromatography gave syn5a (44.6 mg, $0.091 \mathrm{mmol}$ ); yield 85\%; ratio syn/anti $=>20: 1$; white solid; $\mathrm{mp} 115-132{ }^{\circ} \mathrm{C} ; R_{\mathrm{f}}=0.50$ (1:6 ethyl acetate/ hexanes); $[\alpha]_{\mathrm{D}}^{25}+40.1$ (c 1.0, $\mathrm{CHCl}_{3}$ ); IR (neat) 698, 777, 836, 937, 1110, 1161, 1256, 1279, 1638, 2855, 2928, 2955, $3387 \mathrm{~cm}^{-1} ;{ }^{1} \mathrm{H} \mathrm{NMR}\left(\mathrm{CDCl}_{3}, 700 \mathrm{MHz}\right) \delta 0.02-0.03(\mathrm{~m}, 3 \mathrm{H})$, 0.06-0.07 (m, 3H), 0.12-0.15 (m, 6H), 0.86-0.88 (m, 18H), 3.04 (d, $J=2.1 \mathrm{~Hz}, 1 \mathrm{H}), 3.45(\mathrm{dd}, J=10.8,8.2 \mathrm{~Hz}, 1 \mathrm{H}), 3.71(\mathrm{~m}, 1 \mathrm{H})$, $3.97(\mathrm{dd}, J=10.3,3.9 \mathrm{~Hz}, 1 \mathrm{H}), 4.05(\mathrm{dd}, J=5.6,2.2 \mathrm{~Hz}, 1 \mathrm{H})$, $4.30(\mathrm{t}, J=2.0 \mathrm{~Hz}, 1 \mathrm{H}), 4.57(\mathrm{~d}, J=1.7 \mathrm{~Hz}, 1 \mathrm{H}), 5.36(\mathrm{dt}, J=$ 10.6, 1.3 Hz, 1H), 5.53 (dt, $J=17.2,1.3 \mathrm{~Hz}, 1 \mathrm{H}), 5.97-6.02(\mathrm{~m}$, 
1H), $7.38(\mathrm{t}, J=7.5 \mathrm{~Hz}, 2 \mathrm{H}), 7.42-7.45(\mathrm{~m}, 1 \mathrm{H}), 7.91-7.93(\mathrm{~m}$, $2 \mathrm{H}) ;{ }^{13} \mathrm{C}$ NMR $\left(175 \mathrm{MHz}, \mathrm{CDCl}_{3}\right) \delta-5.5,-5.4,-4.5,-4.1,18.0$, 18.2, 25.7, 25.7, 25.8, 25.9, 61.1, 64.7, 65.5, 72.6, 76.1, 118.0, $127.3,128.1,130.5,133.6,135.4,155.5$; HRMS (FAB) $m / z:[\mathrm{M}+$ $\mathrm{H}]^{+}$calcd for $\mathrm{C}_{26} \mathrm{H}_{46} \mathrm{O}_{4} \mathrm{NSi}_{2}$ 492.2965, found 492.2962.

(R)-1-((4R,5R,6R)-5-((tert-Butyldimethylsilyl)oxy)-4-(( tertbutyldimethylsilyl)oxy)methyl)-2-phenyl-5,6-dihydro-4H-1,3oxazin-6-yl)prop-2-en-1-ol (anti-5a). Yield 85\%; ratio syn/anti $=>20: 1$; white solid; $\mathrm{mp} 106-110{ }^{\circ} \mathrm{C} ; R_{\mathrm{f}}=0.53$ (1:6 ethyl acetate-hexanes); $[\alpha]_{\mathrm{D}}^{25}+21.6\left(c 0.6, \mathrm{CHCl}_{3}\right)$; IR (neat) 699, 778, 836, 937, 1033, 1118, 1256, 1280, 1651, 2934, 2951, $3358 \mathrm{~cm}^{-1}$; ${ }^{1} \mathrm{H}$ NMR $\left(\mathrm{CDCl}_{3}, 500 \mathrm{MHz}\right) \delta 0.03(\mathrm{~s}, 3 \mathrm{H}), 0.07(\mathrm{~s}, 3 \mathrm{H}), 0.16(\mathrm{~s}$, $3 \mathrm{H}), 0.17(\mathrm{~s}, 3 \mathrm{H}), 0.87(\mathrm{~s}, 9 \mathrm{H}), 0.89(\mathrm{~s}, 9 \mathrm{H}), 2.74(\mathrm{~d}, J=5.0 \mathrm{~Hz}$, $1 \mathrm{H}), 3.58$ (dd, $J=10.5,7.0 \mathrm{~Hz}, 1 \mathrm{H}), 3.67-3.70$ (m, 1H), 3.96 (dd, $J=10.5,3.5 \mathrm{~Hz}, 1 \mathrm{H}), 4.05(\mathrm{dd}, J=8.0,2.5 \mathrm{~Hz}, 1 \mathrm{H}), 4.47(\mathrm{t}, J=$ $2.5 \mathrm{~Hz}, 1 \mathrm{H}), 4.51-4.56(\mathrm{~m}, 1 \mathrm{H}), 5.33(\mathrm{dt}, J=12.0,1.5 \mathrm{~Hz}, 1 \mathrm{H})$, $5.45(\mathrm{dt}, J=19.0,1.5 \mathrm{~Hz}, 1 \mathrm{H}), 7.23-7.43(\mathrm{~m}, 3 \mathrm{H}), 7.90-7.92(\mathrm{~m}$, $2 \mathrm{H}) ;{ }^{13} \mathrm{C} \mathrm{NMR}\left(125 \mathrm{MHz}, \mathrm{CDCl}_{3}\right) \delta-5.2,-4.3,-4.2,18.2,18.4$, 26.0, 26.1, 60.8, 64.6, 71.6, 75.1, 75.4, 77.4, 117.1, 127.6, 128.2, $130.7,133.7,137.8,155.4$; HRMS (FAB) $m / z:[\mathrm{M}+\mathrm{H}]^{+}$calcd for $\mathrm{C}_{26} \mathrm{H}_{46} \mathrm{O}_{4} \mathrm{NSi}_{2}$ 492.2965, found 492.2963.

(S)-1-((4R,5R,6R)-5-((tert-Butyldimethylsilyl)oxy)-4-((tertbutyldimethylsilyl)oxy)methyl)-2-phenyl-5,6-dihydro-4H-1,3oxazin-6-yl)ethanol (syn-5b). Oxazine 4 (50 mg, $0.108 \mathrm{mmol})$ was dissolved in dry methanol $(2.16 \mathrm{~mL})$ and cooled to $-78{ }^{\circ} \mathrm{C}$. Ozone was then passed through the solution until the reaction was complete. The reaction mixture was quenched with methyl sulfide $(0.08 \mathrm{~mL})$ and allowed to warm to $\mathrm{rt}$. The solvent was evaporated under reduced pressure, and the crude aldehyde was immediately employed in the next step without further purification. Zinc chloride (1.0 M solution in diethyl ether, $0.11 \mathrm{~mL}, 0.119 \mathrm{mmol}$ ) was slowly added to a solution of the aldehyde in THF $(1.08 \mathrm{~mL})$ at $-78{ }^{\circ} \mathrm{C}$ and stirred for $0.5 \mathrm{~h}$. Methylmagnesium bromide (3.0 $\mathrm{M}$ solution in diethyl ether, $0.11 \mathrm{~mL}, 0.324 \mathrm{mmol}$ ) was added to this solution at $-78{ }^{\circ} \mathrm{C}$ and allowed to react for $1 \mathrm{~h}$. The reaction was quenched with saturated $\mathrm{NH}_{4} \mathrm{Cl}$, and warmed to rt. The organic layer was separated and the aqueous layer was extracted with ethyl acetate. The combined organic layer was washed with saturated $\mathrm{NaHCO}_{3}$ and brine, dried with $\mathrm{MgSO}_{4}$, and filtered. The filtrate was concentrated in vacuo. Purification using silica gel column chromatography gave syn-5b $(21.0 \mathrm{mg}, 0.044 \mathrm{mmol})$ : yield $59 \%$; ratio syn/anti $=2.2: 1$; colorless liquid; $R_{\mathrm{f}}=0.4$ (1: 6 ethyl acetate-hexanes); $[\alpha]_{\mathrm{D}}^{25}+3.7\left(c 1.0, \mathrm{CHCl}_{3}\right)$; IR (neat) 667, 777, $837,1071,1111,1141,1257,1653,2858,2930,2955$, $3385 \mathrm{~cm}^{-1} ;{ }^{1} \mathrm{H} \mathrm{NMR}\left(\mathrm{CDCl}_{3}, 700 \mathrm{MHz}\right) \delta 0.05(\mathrm{~s}, 3 \mathrm{H}), 0.08(\mathrm{~s}$, $3 \mathrm{H}), 0.13(\mathrm{~s}, 6 \mathrm{H}), 0.81-0.94(\mathrm{~m}, 18 \mathrm{H}), 2.94(\mathrm{~s}, 1 \mathrm{H}), 3.44(\mathrm{dd}, J=$ 10.5, 8.4 Hz, 1H), $3.70(\mathrm{~m}, 1 \mathrm{H}), 3.92(\mathrm{dd}, J=6.0,0.9 \mathrm{~Hz}, 1 \mathrm{H})$, $3.98(\mathrm{dd}, J=10.5,4.0 \mathrm{~Hz}, 1 \mathrm{H}), 4.20(\mathrm{t}, J=6.2 \mathrm{~Hz}, 1 \mathrm{H}), 4.28(\mathrm{t}$, $J=1.6 \mathrm{~Hz}, 1 \mathrm{H}), 7.36-7.39$ (m, 2H), 7.41-7.43 (m, 1H), 7.91-7.93 $(\mathrm{m}, 2 \mathrm{H}) ;{ }^{13} \mathrm{C} \mathrm{NMR}\left(\mathrm{CDCl}_{3}, 175 \mathrm{MHz}\right) \delta-5.3,-5.2,-3.9,18.2$, 18.3, 18.4, 61.4, 65.1, 65.8, 67.9, 127.5, 128.2, 130.7, 134.0, 155.8; HRMS (FAB) $m / z$ : $[\mathrm{M}+\mathrm{H}]^{+}$calcd for $\mathrm{C}_{25} \mathrm{H}_{46} \mathrm{O}_{4} \mathrm{NSi}_{2}$ 480.2965 , found 480.2964 .

(R)-1-((4R,5R,6R)-5-((tert-Butyldimethylsilyl)oxy)-4-((tertbutyldimethylsilyl)oxy)methyl)-2-phenyl-5,6-dihydro-4H-1,3- oxazin-6-yl)ethanol (anti-5b). Yield 59\%; ratio syn/anti = $2.2: 1$; white solid; mp 122-124 ${ }^{\circ} \mathrm{C} ; R_{\mathrm{f}}=0.45$ (1:6 ethyl acetate-hexanes); $[\alpha]_{\mathrm{D}}^{25}+2.8\left(c\right.$ 1.0, $\mathrm{CHCl}_{3}$ ); IR (neat) 702, 777, 838, 1103, 1140, 1363, 1648, 2856, 2929, 2978, $3193 \mathrm{~cm}^{-1} ;{ }^{1} \mathrm{H}$ NMR $\left(\mathrm{CDCl}_{3}, 700 \mathrm{MHz}\right) \delta 0.04(\mathrm{~s}, 3 \mathrm{H}), 0.09(\mathrm{~s}, 3 \mathrm{H}), 0.17$ (s, $6 \mathrm{H}), 0.87-0.90(\mathrm{~m}, 18 \mathrm{H}), 1.40(\mathrm{~d}, J=6.2 \mathrm{~Hz}, 3 \mathrm{H}), 2.53(\mathrm{~s}, 1 \mathrm{H})$, $3.63-3.69(\mathrm{~m}, 2 \mathrm{H}), 3.92(\mathrm{dd}, J=8.1,2.5 \mathrm{~Hz}, 1 \mathrm{H}), 3.97(\mathrm{dd}, J=$ 10.1, $3.0 \mathrm{~Hz}, 1 \mathrm{H}), 4.15-4.18(\mathrm{~m}, 1 \mathrm{H}), 4.63(\mathrm{dd}, J=3.2,2.9 \mathrm{~Hz}$, 1H), 7.33-7.38 (m, 2H), 7.40-7.43 (m, 1H), 7.91-7.93 (m, 2H); ${ }^{13} \mathrm{C} \mathrm{NMR}\left(\mathrm{CDCl}_{3}, 175 \mathrm{MHz}\right) \delta-5.1,-4.4,-4.3,18.2,18.5,20.6$, 26.0, 60.6, 64.5, 66.6, 127.6, 128.2, 130.6, 133.8, 155.2; HRMS (FAB) $m / z:[\mathrm{M}+\mathrm{H}]^{+}$calcd for $\mathrm{C}_{25} \mathrm{H}_{46} \mathrm{O}_{4} \mathrm{NSi}_{2} 480.2965$, found 480.2961 .

$(S)-1-((4 R, 5 R, 6 R)-5-(($ tert-Butyldimethylsilyl)oxy)-4-((tertbutyldimethylsilyl)oxy)methyl)-2-phenyl-5,6-dihydro-4H-1,3oxazin-6-yl)propan-1-ol $($ syn-5c). Yield $60 \%$; ratio syn/anti $=$ $14: 1$; colorless liquid; $R_{\mathrm{f}}=0.5$ (1:6 ethyl acetate-hexanes); $[\alpha]_{\mathrm{D}}^{25}+3.9\left(c 1.0, \mathrm{CHCl}_{3}\right)$; IR (neat) 698, 777, 836, 1112, 1257, 1653, 2858, 2930, 2956, $3385 \mathrm{~cm}^{-1}$; ${ }^{1} \mathrm{H} \mathrm{NMR}\left(\mathrm{CDCl}_{3}, 700 \mathrm{MHz}\right)$ $\delta$ 0.04-0.15 (m, 12H), 0.81-0.94 (m, 18H), $1.07(\mathrm{t}, J=7.4 \mathrm{~Hz}$, $3 \mathrm{H}), 1.63-1.68(\mathrm{~m}, 1 \mathrm{H}), 1.71-1.74(\mathrm{~m}, 1 \mathrm{H}), 3.02(\mathrm{~s}, 1 \mathrm{H}), 3.47$ (dd, $J=10.5,8.3 \mathrm{~Hz}, 1 \mathrm{H}), 3.68-3.71(\mathrm{~m}, 1 \mathrm{H}), 3.93-3.99(\mathrm{~m}, 2 \mathrm{H})$, $4.04(\mathrm{dd}, J=5.2,0.4 \mathrm{~Hz}, 1 \mathrm{H}), 4.30(\mathrm{t}, J=1.7 \mathrm{~Hz}, 1 \mathrm{H}), 7.35-7.38$ $(\mathrm{m}, 2 \mathrm{H}), 7.41-7.43(\mathrm{~m}, 1 \mathrm{H}), 7.91-7.93(\mathrm{~m}, 2 \mathrm{H}) ;{ }^{13} \mathrm{C} \mathrm{NMR}$ $\left(\mathrm{CDCl}_{3}, 175 \mathrm{MHz}\right) \delta-5.3,-5.2,-4.7,-3.9,9.9,10.1,18.2,25.5$, 26.1, 61.5, 65.1, 66.3, 73.1, 75.6, 127.5, 128.2, 130.7, 134.0, 155.9; HRMS (FAB) $m / z$ : $[\mathrm{M}+\mathrm{H}]^{+}$calcd for $\mathrm{C}_{26} \mathrm{H}_{48} \mathrm{O}_{4} \mathrm{NSi}_{2}$ 494.3122, found 494.3120.

$(R)-1-((4 R, 5 R, 6 R)-5-(($ tert-Butyldimethylsilyl)oxy)-4-((tertbutyldimethylsilyl)oxy)methyl)-2-phenyl-5,6-dihydro-4H-1,3oxazin-6-yl)propan-1-ol (anti-5c). Yield 60\%; ratio syn/anti = $14: 1$; colorless liquid; $R_{\mathrm{f}}=0.55$ ( $1: 6$ ethyl acetate-hexanes); $[\alpha]_{\mathrm{D}}^{25}+3.2\left(c 1.0, \mathrm{CHCl}_{3}\right)$; IR (neat) 697, 778, 836, 1140, 1256, 1655, 2858, 2930, 2956, $3385 \mathrm{~cm}^{-1} ;{ }^{1} \mathrm{H} \mathrm{NMR}\left(\mathrm{CDCl}_{3}, 700 \mathrm{MHz}\right)$ $\delta 0.04(\mathrm{~s}, 3 \mathrm{H}), 0.08(\mathrm{~s}, 3 \mathrm{H}), 0.16-0.17(\mathrm{~m}, 6 \mathrm{H}), 0.87-0.90(\mathrm{~m}$, $18 \mathrm{H}), 1.07(\mathrm{t}, J=7.4 \mathrm{~Hz}, 3 \mathrm{H}), 1.60-1.64(\mathrm{~m}, 1 \mathrm{H}), 1.87-1.91(\mathrm{~m}$, $1 \mathrm{H}), 2.53(\mathrm{~s}, 1 \mathrm{H}), 3.63-3.65(\mathrm{~m}, 1 \mathrm{H}), 3.67-3.69(\mathrm{~m}, 1 \mathrm{H})$, 3.96-3.98 (m, 2H), 4.00-4.03 (m, 1H), 4.45 (dd, $J=3.3,2.6 \mathrm{~Hz}$, 1H), 7.35-7.38 (m, 2H), 7.40-7.42 (m, 1H), 7.90-7.93 (m, 2H); ${ }^{13} \mathrm{C} \mathrm{NMR}\left(\mathrm{CDCl}_{3}, 175 \mathrm{MHz}\right) \delta-5.1,-4.3,-4.2,9.5,18.2,18.5$, 64.6, 71.4, 75.3, 127.6, 128.2, 130.6, 133.8, 155.3; HRMS (FAB) $m / z:[\mathrm{M}+\mathrm{H}]^{+}$calcd for $\mathrm{C}_{26} \mathrm{H}_{48} \mathrm{O}_{4} \mathrm{NSi}_{2}$ 494.3122, found 494.3120.

$(S)-1-((4 R, 5 R, 6 R)-5-(($ tert-Butyldimethylsilyl)oxy)-4-(( tertbutyldimethylsilyl)oxy)methyl)-2-phenyl-5,6-dihydro-4H-1,3oxazin-6-yl)but-3-en-1-ol (syn-5d). Yield 60\%; ratio syn/anti = $>20$ : 1 ; colorless liquid; $R_{\mathrm{f}}=0.5$ ( $1: 6$ ethyl acetate-hexanes); $[\alpha]_{\mathrm{D}}^{25}+4.6\left(c\right.$ 1.0, $\left.\mathrm{CHCl}_{3}\right)$; IR (neat) 777, 837, 1007, 1033, 1057, 1110, 1257, 1655, 2859, 2930, 2955, $3419 \mathrm{~cm}^{-1} ;{ }^{1} \mathrm{H}$ NMR $\left(\mathrm{CDCl}_{3}, 700 \mathrm{MHz}\right) \delta 0.03-0.08(\mathrm{~m}, 6 \mathrm{H}), 0.15(\mathrm{~s}, 6 \mathrm{H}), 0.80-0.95$ $(\mathrm{m}, 18 \mathrm{H}), 2.42-2.45(\mathrm{~m}, 1 \mathrm{H}), 2.50-2.53(\mathrm{~m}, 1 \mathrm{H}), 3.12(\mathrm{~s}, 1 \mathrm{H})$, 3.47 (dd, $J=10.4,8.2 \mathrm{~Hz}, 1 \mathrm{H}), 3.69-3.71(\mathrm{~m}, 1 \mathrm{H}), 3.97$ (dd, $J=$ 10.5, 3.8 Hz, 1H), 4.08-4.13 (m, 2H), 4.32 (s, 1H), 5.14-5.19 (m, $1 \mathrm{H}), 5.21$ (d, $J=1.61 \mathrm{~Hz}, 1 \mathrm{H}), 5.92-6.00(\mathrm{~m}, 1 \mathrm{H}), 7.35-7.39(\mathrm{~m}$, $2 \mathrm{H}), 7.41-7.44(\mathrm{~m}, 1 \mathrm{H}), 7.92-7.95(\mathrm{~m}, 2 \mathrm{H}) ;{ }^{13} \mathrm{C} \mathrm{NMR}\left(\mathrm{CDCl}_{3}\right.$, $175 \mathrm{MHz}) \delta-5.3,-5.2,-4.4,-3.9,18.2,18.4,25.9,26.1,37.3$, 
61.3, 66.5, 71.4, 74.8, 118.2, 127.6, 128.2, 128.4, 130.7, 134.0, 134.3, 155.9; HRMS (FAB) $m / z:[\mathrm{M}+\mathrm{H}]^{+}$calcd for $\mathrm{C}_{27} \mathrm{H}_{48} \mathrm{O}_{4} \mathrm{NSi}_{2}$ 506.3122 , found 506.3121 .

(R)-1-((4R,5R,6R)-5-((tert-Butyldimethylsilyl)oxy)-4-((tertbutyldimethylsilyl)oxy)methyl)-2-phenyl-5,6-dihydro-4H-1,3oxazin-6-yl)but-3-en-1-ol (anti-5d). Yield 60\%; ratio syn/anti $=>20: 1$; colorless liquid; $R_{\mathrm{f}}=0.55$ (1:6 ethyl acetatehexanes); $[\alpha]_{\mathrm{D}}^{25}+4.0$ (c 1.0, $\mathrm{CHCl}_{3}$ ); IR (neat) 697, 778, 837, 1072, 1119, 1257, 1656, 2858, 2929, 2955, $3356 \mathrm{~cm}^{-1} ;{ }^{1} \mathrm{H}$ NMR $\left(\mathrm{CDCl}_{3}, 700 \mathrm{MHz}\right) \delta 0.04(\mathrm{~s}, 3 \mathrm{H}), 0.08(\mathrm{~s}, 3 \mathrm{H}), 0.15(\mathrm{~s}, 6 \mathrm{H})$, 0.80-0.98 (m, 18H), 2.37-2.41 (m, 2H), 2.67-2.69 (m, 1H), 3.60 (dd, $J=10.4,7.1 \mathrm{~Hz}, 1 \mathrm{H}), 3.67-3.90(\mathrm{~m}, 1 \mathrm{H}), 3.97$ (dd, $J=10.4$, $3.4 \mathrm{~Hz}, 1 \mathrm{H}), 4.01$ (dd, $J=8.3,1.9 \mathrm{~Hz}, 1 \mathrm{H}), 4.05-4.08(\mathrm{~m}, 1 \mathrm{H})$, $4.45(\mathrm{t}, J=2.4 \mathrm{~Hz}, 1 \mathrm{H}), 5.20-5.26(\mathrm{~m}, 2 \mathrm{H}), 5.90-6.00(\mathrm{~m}, 1 \mathrm{H})$, 7.35-7.39 (m, 2H), 7.40-7.43 (m, 1H), 7.91-7.93 (m, 2H); ${ }^{13} \mathrm{C}$ NMR $\left(\mathrm{CDCl}_{3}, 175 \mathrm{MHz}\right) \delta-5.2,-4.4,-4.3,9.7,13.9,18.2,18.4$, 26.2 , 38.4, 61.0, 64.4, 64.7, 68.7, 75.2, 119.0, 127.5, 128.2, 130.6, 133.9, 134.3, 155.2; HRMS (FAB) $m / z:[\mathrm{M}+\mathrm{H}]^{+}$calcd for $\mathrm{C}_{27} \mathrm{H}_{48} \mathrm{O}_{4} \mathrm{NSi}_{2}$ 506.3122, found 506.3120.

(S)-1-((4R,5R,6R)-5-((tert-Butyldimethylsilyl)oxy)-4-((tertbutyldimethylsilyl)oxy)methyl)-2-phenyl-5,6-dihydro-4H-1,3oxazin-6-yl)pent-4-en-1-ol (syn-5e). Yield 62\%; ratio syn/anti $=>20: 1$; colorless liquid; $R_{\mathrm{f}}=0.5$ ( $1: 6$ ethyl acetate-hexanes); $[\alpha]_{\mathrm{D}}^{25}+10.0\left(c 1.0, \mathrm{CHCl}_{3}\right)$; IR (neat) 698, 778, 837, 1071, 1111, 1257, 1655, 2858, 2930, 2954, $3423 \mathrm{~cm}^{-1} ;{ }^{1} \mathrm{H}$ NMR $\left(\mathrm{CDCl}_{3}\right.$, $700 \mathrm{MHz}) \delta-0.07(\mathrm{~s}, 2 \mathrm{H}),-0.03(\mathrm{~s}, 2 \mathrm{H}), 0.00(\mathrm{~s}, 2 \mathrm{H}), 0.67(\mathrm{~d}, J=$ $5.2 \mathrm{~Hz}, 6 \mathrm{H}), 0.79(\mathrm{~s}, 9 \mathrm{H}), 0.81(\mathrm{~s}, 9 \mathrm{H}), 1.65-1.67(\mathrm{~m}, 2 \mathrm{H})$, 2.14-2.17 (m, 1H), 2.27-2.31 (m, 1H), 3.02 (s, 1H), 3.62-3.63 (m, 1H), $3.90(\mathrm{dd}, J=10.5,3.9 \mathrm{~Hz}, 1 \mathrm{H}), 3.97$ (s, 2H), 4.23 (s, $1 \mathrm{H}), 4.94(\mathrm{~d}, J=11.3 \mathrm{~Hz}, 1 \mathrm{H}), 5.02(\mathrm{~d}, J=20.4 \mathrm{~Hz}, 1 \mathrm{H})$, 5.79-5.84 (m, 1H), $7.30(\mathrm{t}, J=9.0 \mathrm{~Hz}, 2 \mathrm{H}), 7.36(\mathrm{t}, J=8.0 \mathrm{~Hz}$, $1 \mathrm{H}), 7.85(\mathrm{~d}, J=7.1 \mathrm{~Hz}, 2 \mathrm{H}) ;{ }^{13} \mathrm{C} \mathrm{NMR}\left(\mathrm{CDCl}_{3}, 175 \mathrm{MHz}\right) \delta-5.5$, $-5.4,-4.7,-4.1,17.9,18.2,25.6,25.7,29.6,31.7,61.1,64.8$, 66.1, 71.1, 75.5, 115.0, 127.3, 128.1, 130.5, 133.7, 138.2, 155.7; HRMS (FAB) $m / z:[\mathrm{M}+\mathrm{H}]^{+}$calcd for $\mathrm{C}_{28} \mathrm{H}_{50} \mathrm{O}_{4} \mathrm{NSi}_{2}$ 520.3278, found 520.3275 .

(R)-1-((4R,5R,6R)-5-((tert-Butyldimethylsilyl)oxy)-4-((tertbutyldimethylsilyl)oxy)methyl)-2-phenyl-5,6-dihydro-4H-1,3oxazin-6-yl)pent-4-en-1-ol (anti-5e). Yield 62\%; ratio syn/anti $=>20: 1$; colorless liquid; $R_{\mathrm{f}}=0.55$ (1:6 ethyl acetatehexanes); $[\alpha]_{\mathrm{D}}^{25}+3.8$ (c 1.0, $\mathrm{CHCl}_{3}$ ); IR (neat) 698, 778, 836, 1117, 1256, 1654, 2858, 2930, 2954, $3384 \mathrm{~cm}^{-1} ;{ }^{1} \mathrm{H}$ NMR $\left(\mathrm{CDCl}_{3}, 700 \mathrm{MHz}\right) \delta 0.04(\mathrm{~s}, 3 \mathrm{H}), 0.08(\mathrm{~s}, 3 \mathrm{H}), 0.16(\mathrm{~s}, 6 \mathrm{H})$, 0.80-0.94 (s, 18H), 1.65-1.71 (m, 1H), 1.95-1.99 (m, 1H), $2.22-2.27(\mathrm{~m}, 1 \mathrm{H}), 2.34-2.38(\mathrm{~m}, 1 \mathrm{H}), 2.51(\mathrm{~s}, 1 \mathrm{H}), 3.62(\mathrm{dd}, J=$ 10.3, $6.8 \mathrm{~Hz}, 1 \mathrm{H}), 3.68(6, J=3.4 \mathrm{~Hz}, 1 \mathrm{H}), 3.97$ (dd, $J=10.3,3.4$ $\mathrm{Hz}, 1 \mathrm{H}), 3.99-4.01(\mathrm{~m}, 1 \mathrm{H}), 4.01-4.06(\mathrm{~m}, 1 \mathrm{H}), 4.45(\mathrm{~s}, 1 \mathrm{H})$, 5.00 (dd, $J=10.1,1.8 \mathrm{~Hz}, 1 \mathrm{H}), 5.08$ (dd, $J=17.2,1.8 \mathrm{~Hz}, 1 \mathrm{H}$ ), 5.88-5.91 (m, 1H), 7.35-7.37 (m, 2H), 7.37-7.40 (m, 1H), 7.90-7.93 (m, 2H); ${ }^{13} \mathrm{C} \mathrm{NMR}\left(\mathrm{CDCl}_{3}, 175 \mathrm{MHz}\right) \delta-5.2,-5.1$, $4.4,-4.3,18.2,18.5,26.0,29.6,33.1,60.7,64.6,69.7,75.7$, 115.2, 127.6, 128.2, 130.7, 133.8, 138.7, 155.3; HRMS (FAB) $m / z$ : $[\mathrm{M}+\mathrm{H}]^{+}$calcd for $\mathrm{C}_{28} \mathrm{H}_{50} \mathrm{O}_{4} \mathrm{NSi}_{2} 520.3278$, found 520.3282 .

(S)-((4R,5R,6R)-5-((tert-Butyldimethylsilyl)oxy)-4-(((tert-butyldimethylsilyl)oxy)methyl)-2-phenyl-5,6-dihydro-4H-1,3-oxazin-6-yl)(phenyl)methanol (syn-5f). Yield 65\%; ratio syn/anti $=8: 1$; col- orless liquid; $R_{\mathrm{f}}=0.57$ (1:6 ethyl acetate-hexanes); $[\alpha]_{\mathrm{D}}^{25}+5.5$ (c 1.0, $\mathrm{CHCl}_{3}$ ); IR (neat) 699, 778, 837, 1112, 1134, 1256, 1472, 1648, 2857, 2929, 2955, $3284 \mathrm{~cm}^{-1}$; ${ }^{1} \mathrm{H} \mathrm{NMR}\left(\mathrm{CDCl}_{3}, 700 \mathrm{MHz}\right)$ $\delta-0.07$ (s, 3H), $0.00(\mathrm{~s}, 3 \mathrm{H}), 0.12(\mathrm{~s}, 6 \mathrm{H}), 0.80(\mathrm{~s}, 9 \mathrm{H}), 0.90(\mathrm{~s}$, 9H), 3.37-3.41 (m, 2H), 3.73 (dd, $J=8.1,3.7 \mathrm{~Hz}, 1 \mathrm{H}), 3.93$ (dd, $J=10.4,3.9 \mathrm{~Hz}, 1 \mathrm{H}), 4.16(\mathrm{~d}, \mathrm{~J}=1.3 \mathrm{~Hz}, 1 \mathrm{H}), 4.28(\mathrm{~d}, J=5.4 \mathrm{~Hz}$, $1 \mathrm{H}), 5.11$ (d, $J=5.5 \mathrm{~Hz}, 1 \mathrm{H}), 7.33-7.44(\mathrm{~m}, 6 \mathrm{H}), 7.50-7.53(\mathrm{~m}$, $2 \mathrm{H}), 7.88-7.92(\mathrm{~m}, 2 \mathrm{H}) ;{ }^{13} \mathrm{C} \mathrm{NMR}\left(\mathrm{CDCl}_{3}, 175 \mathrm{MHz}\right) \delta-5.7,-$ 5.5, -4.4, -3.9, 18.1, 25.8, 61.1, 64.7, 65.9, 74.3, 126.0, 127.4, 128.1, 128.3, 128.4, 128.5, 130.5, 133.7, 139.7, 155.7; HRMS (FAB) $m / z:[\mathrm{M}+\mathrm{H}]^{+}$calcd for $\mathrm{C}_{30} \mathrm{H}_{48} \mathrm{O}_{4} \mathrm{NSi}_{2}$ 542.3122, found 542.3121 .

$(R)-((4 R, 5 R, 6 R)-5-(($ tert-Butyldimethylsilyl)oxy)-4-((tertbutyldimethylsilyl)oxy)methyl)-2-phenyl-5,6-dihydro-4H-1,3oxazin-6-yl)(phenyl)methanol (anti-5f). Yield 65\%; ratio syn/ anti $=8: 1$; white solid; $\mathrm{mp} 145-155{ }^{\circ} \mathrm{C} ; R_{\mathrm{f}}=0.48$ (1:6 ethyl acetate-hexanes); $[\alpha]_{\mathrm{D}}^{25}+2.5$ (c 1.0, $\mathrm{CHCl}_{3}$ ); IR (neat) 699, 775, 830, 1016, 1033, 1057, 2858, 2925, 2950, $3211 \mathrm{~cm}^{-1} ;{ }^{1} \mathrm{H}$ NMR $\left(\mathrm{CDCl}_{3}, 700 \mathrm{MHz}\right) \delta-0.06(\mathrm{~s}, 3 \mathrm{H}), 0.00(\mathrm{~s}, 3 \mathrm{H}), 0.10-0.25(\mathrm{~m}$, $6 \mathrm{H}), 0.70-0.95(\mathrm{~m}, 18 \mathrm{H}), 3.09(\mathrm{~s}, 1 \mathrm{H}), 3.53(\mathrm{dd}, J=10.2,6.9 \mathrm{~Hz}$, $1 \mathrm{H}), 3.65-3.69$ (m, 1H), 3.90 (dd, $J=10.4,3.4 \mathrm{~Hz}, 1 \mathrm{H}), 4.17$ (dd, $J=7.9,2.1 \mathrm{~Hz}, 1 \mathrm{H}), 4.44(\mathrm{t}, J=2.7 \mathrm{~Hz}, 1 \mathrm{H}), 5.00(\mathrm{dd}, J=7.9,2.8$ $\mathrm{Hz}, 1 \mathrm{H}), 7.20-7.40(\mathrm{~m}, 8 \mathrm{H}), 7.61-7.65(\mathrm{~m}, 2 \mathrm{H})$; ${ }^{13} \mathrm{C} \mathrm{NMR}$ $\left(\mathrm{CDCl}_{3}, 175 \mathrm{MHz}\right) \delta-5.3,-5.0,-4.5,-4.3,18.5,26.0,60.8,64.6$, 64.8, 73.00, 76.4, 127.1, 127.4, 127.6, 127.7, 127.9, 128.1, 128.2, $128.5,128.6,130.5,133.5,141.6,155.3$; HRMS (FAB) $m / z:[\mathrm{M}+$ $\mathrm{H}]^{+}$calcd for $\mathrm{C}_{30} \mathrm{H}_{48} \mathrm{O}_{4} \mathrm{NSi}_{2} 542.3122$, found 542.3124 .

(5S,6S,7R,8R)-6-(Benzoyloxy)-8-(((benzyloxy)carbonyl)amino)7,9-bis((tert-butyldimethylsilyl)oxy)non-1-en-5-yl methanesulfonate (8). Triethylamine $(74 \mu \mathrm{L}, 0.533 \mathrm{mmol})$ and $\mathrm{MsCl}$ (56 $\mu \mathrm{L}, 0.727 \mathrm{mmol}$ ) were successively added to a solution of pentenyl alcohol syn-5e (126 mg, $0.242 \mathrm{mmol})$ in dichloromethane $(4.84 \mathrm{~mL})$ at $0{ }^{\circ} \mathrm{C}$. The reaction mixture was stirred at $0{ }^{\circ} \mathrm{C}$ for $1 \mathrm{~h}$, and then distilled water was added. The organic layer was separated and the aqueous layer was extracted with dichloromethane. The combined organic layer was washed with saturated $\mathrm{NH}_{4} \mathrm{Cl}$, saturated $\mathrm{NaHCO}_{3}$, and brine, dried with $\mathrm{MgSO}_{4}$, and filtered. The filtrate was concentrated in vacuo. Purification using silica gel column chromatography gave the secondary mesylate intermediate $(133 \mathrm{mg}$, $0.223 \mathrm{mmol}$ ); colorless liquid; $R_{\mathrm{f}}=0.48$ (1:6 ethyl acetatehexanes); $[\alpha]_{\mathrm{D}}^{25}+19.3\left(c\right.$ 1.0, $\mathrm{CHCl}_{3}$ ); IR (neat) 699, 778, 837, 926, 1110, 1178, 1256, 1359, 1658, 2858, 2930, 2954, $3423 \mathrm{~cm}^{-1} ;{ }^{1} \mathrm{H}$ NMR $\left(\mathrm{CDCl}_{3}, 700 \mathrm{MHz}\right) \delta-0.07$ (s, 2H), -0.04 (s, $2 \mathrm{H}), 0.00(\mathrm{~s}, 2 \mathrm{H}), 0.04(\mathrm{~d}, J=5.2 \mathrm{~Hz}, 6 \mathrm{H}), 0.78(\mathrm{~s}, 9 \mathrm{H}), 0.80(\mathrm{~s}$, $9 \mathrm{H}), 1.71-1.74(\mathrm{~m}, 1 \mathrm{H}), 1.88-1.92(\mathrm{~m}, 1 \mathrm{H}), 2.21-2.25(\mathrm{~m}, 2 \mathrm{H})$, $2.98(\mathrm{~s}, 3 \mathrm{H}), 3.42(\mathrm{dd}, J=10.5,7.9 \mathrm{~Hz}, 1 \mathrm{H}), 3.63-3.65(\mathrm{~m}, 1 \mathrm{H})$, $3.90(\mathrm{dd}, J=10.6,3.8 \mathrm{~Hz}, 1 \mathrm{H}), 4.17$ (s, 1H), 4.26 (dd, $J=8.5,0.7$ $\mathrm{Hz}, 1 \mathrm{H}), 4.96$ (d, $J=10.2 \mathrm{~Hz}, 1 \mathrm{H}), 5.00-5.04(\mathrm{~m}, 2 \mathrm{H}), 5.75-5.79$ $(\mathrm{m}, 1 \mathrm{H}), 7.30(\mathrm{t}, J=7.9 \mathrm{~Hz}, 2 \mathrm{H}), 7.35(\mathrm{t}, J=8.0 \mathrm{~Hz}, 1 \mathrm{H}), 7.79(\mathrm{~d}$, $J=8.5 \mathrm{~Hz}, 2 \mathrm{H}) ;{ }^{13} \mathrm{C} \mathrm{NMR}\left(\mathrm{CDCl}_{3}, 175 \mathrm{MHz}\right) \delta-5.5,-5.4,-4.6$, 3.9, 18.0, 18.1, 25.6, 25.8, 28.4, 29.6, 38.8, 60.6, 64.0, 64.5, 74.9, $82.4,115.7,127.4,128.2,130.5,133.8,136.8,155.9$; HRMS (FAB) $m / z:[\mathrm{M}+\mathrm{H}]^{+}$calcd for $\mathrm{C}_{29} \mathrm{H}_{52} \mathrm{O}_{6} \mathrm{NSi}_{2} \mathrm{~S} 598.3054$, found 598.3055. A solution of $\mathrm{NaHCO}_{3}(0.6 \mathrm{M}$ in distilled water, $1.5 \mathrm{~mL}, 0.892 \mathrm{mmol})$ and benzyl chloroformate $(0.127 \mathrm{~mL}$, 
$0.892 \mathrm{mmol}$ ) was successively added to a solution of this mesylate $(133 \mathrm{mg}, 0.223 \mathrm{mmol})$ in dichloromethane $(1.5 \mathrm{~mL})$. The reaction mixture was stirred at rt for $48 \mathrm{~h}$. The organic layer was separated and the aqueous layer was extracted with dichloromethane. The combined organic layer was washed with distilled water and brine, dried with $\mathrm{MgSO}_{4}$, and filtered. The filtrate was concentrated in vacuo. Purification using silica gel column chromatography gave 8 (125 mg, $0.167 \mathrm{mmol})$; yield $69 \%$ for two steps; rotamer ratio $>20$ : 1 ; colorless liquid; $R_{\mathrm{f}}=0.35$ ( $1: 6$ ethyl acetate-hexanes); $[\alpha]_{\mathrm{D}}^{25}-6.3\left(c 1.0, \mathrm{CHCl}_{3}\right)$; IR (neat) 712, 778, 836, 914, 1033, 1056, 1099, 1175, 1258, 1345, 1722, 2858, 2931, 2954, $3453 \mathrm{~cm}^{-1} ;{ }^{1} \mathrm{H} \mathrm{NMR}\left(\mathrm{CDCl}_{3}, 700 \mathrm{MHz}\right) \delta$ $0.00(\mathrm{~s}, 3 \mathrm{H}), 0.03(\mathrm{~d}, J=6.8 \mathrm{~Hz}, 3 \mathrm{H}), 0.10(\mathrm{~d}, J=15.5 \mathrm{~Hz}, 6 \mathrm{H})$, 0.82 (s, 9H), 0.87 (s, 9H), 1.88-1.90 (m, 1H), 2.00-2.02 (m, 1H), 2.26-2.29 (m, 2H), 2.89 (s, 3H), 3.66 (dd, $J=10.3,5.8 \mathrm{~Hz}, 1 \mathrm{H})$, 3.80-3.83 (m, 1H), 3.89-3.91 (m, 1H), 4.23-4.25 (m, 1H), 5.01 (d, $J=10.4 \mathrm{~Hz}, 1 \mathrm{H}), 5.05(\mathrm{~d}, J=12.2 \mathrm{~Hz}, 1 \mathrm{H}), 5.09-5.12(\mathrm{~m}, 2 \mathrm{H})$, $5.45(\mathrm{t}, J=5.6 \mathrm{~Hz}, 1 \mathrm{H}), 5.81-5.83(\mathrm{~m}, 1 \mathrm{H}), 7.30-7.37(\mathrm{~m}, 6 \mathrm{H})$, 7.54-7.56 (m, 2H), 8.07 (d, $J=7.4 \mathrm{~Hz}, 2 \mathrm{H}) ;{ }^{13} \mathrm{C} \mathrm{NMR}\left(\mathrm{CDCl}_{3}\right.$, $175 \mathrm{MHz}) \delta-5.7,-5.5,-4.8,-4.3,18.0,18.2,25.9,29.1,31.2$, 38.7, 54.6, 61.4, 66.8, 69.4, 74.1, 80.6, 115.7, 127.9, 128.4, 128.6, 129.9, 133.4, 136.5, 136.9, 155.9, 165.4; HRMS (FAB) $m / z$ : $[\mathrm{M}+\mathrm{H}]^{+}$calcd for $\mathrm{C}_{37} \mathrm{H}_{60} \mathrm{O}_{9} \mathrm{NSi}_{2} \mathrm{~S} 750.3527$, found 750.3528 .

$(2 R, 3 R, 4 R, 5 R)$-Benzyl 2-(but-3-en-1-yl)-4-((tert-butyldimethylsilyl)oxy)-5-((tert-butyldimethylsilyl)oxy)methyl)-3-hydroxypyrrolidine-1-carboxylate (7). $\mathrm{NaH}(21.4 \mathrm{mg}, 0.892 \mathrm{mmol})$ was added to a solution of mesylate $8(223 \mathrm{mg}, 0.297 \mathrm{mmol})$ in THF ( $6 \mathrm{~mL})$ at $\mathrm{rt}$. The reaction mixture was stirred for $12 \mathrm{~h}$, and then distilled water was added. The organic layer was separated, and the aqueous layer was extracted with ethyl acetate. The combined organic layer was washed with distilled water and brine, dried with $\mathrm{MgSO}_{4}$, and filtered. The filtrate was concentrated in vacuo. Purification using silica gel column chromatography gave pyrrolidine 7 (135 mg, $0.246 \mathrm{mmol}$ ); yield 83\%; rotamer ratio $3: 1$; colorless liquid; $R_{\mathrm{f}}=0.5$ ( $1: 6$ ethyl acetatehexanes); $[\alpha]_{\mathrm{D}}^{25}+2.8$ (c 0.1, $\mathrm{CHCl}_{3}$ ); IR (neat) 474, 1012, 1032, 1055, 1656, 2825, 2936, 2953, $3420 \mathrm{~cm}^{-1}$; ${ }^{1} \mathrm{H} \mathrm{NMR}\left(\mathrm{CDCl}_{3}\right.$, $700 \mathrm{MHz}) \delta 0.04-0.10(\mathrm{~m}, 12 \mathrm{H}), 0.84-0.90$ (m, 18H), 1.85-1.88 (m, 2H), 2.00-2.15 (m, 2H), 3.61 (dd, $J=10.6,1.3 \mathrm{~Hz}, 1 \mathrm{H})$, 3.70-3.85 (m, 2H), $3.86(\mathrm{~d}, J=2.1 \mathrm{~Hz}, 1 \mathrm{H}), 4.05-4.12(\mathrm{~m}, 2 \mathrm{H})$, 4.49 (dd, $J=10.6,3.0 \mathrm{~Hz}, 0.75 \mathrm{H}), 4.57$ (d, $J=11.3 \mathrm{~Hz}, 0.25 \mathrm{H})$, $4.87(\mathrm{dd}, J=11.9 \mathrm{~Hz}, 0.75 \mathrm{H}), 4.90-4.92(\mathrm{~m}, 0.25 \mathrm{H}), 4.97$ (d, $J=$ $15.9 \mathrm{~Hz}, 0.75 \mathrm{H}), 5.07$ (d, $J=15.9 \mathrm{~Hz}, 0.25 \mathrm{H}), 5.10-5.16(\mathrm{~m}, 2 \mathrm{H})$, 5.65-5.75 (m, 0.75H), 5.80-5.88 (m, 0.25H), 7.30-7.38 (m, 5H); ${ }^{13} \mathrm{C} \mathrm{NMR}\left(\mathrm{CDCl}_{3}, 175 \mathrm{MHz}\right) \delta-5.8,-5.0,17.9,18.3,25.7,30.7$, $31.1,61.4,62.8,66.7,68.5,68.7,114.7,127.9,128.2,128.3$, 128.5, 128.6, 136.5, 136.7, 137.9, 138.0, 154.2, 154.7; HRMS (FAB) $m / z:[\mathrm{M}+\mathrm{H}]^{+} \mathrm{C}_{29} \mathrm{H}_{52} \mathrm{O}_{5} \mathrm{NSi}_{2}$ calcd for 550.3384 , found 550.3383 .

$(1 R, 2 R, 3 R, 7 \mathrm{a} R)-2-(($ tert-Butyldimethylsilyl)oxy)-3-((tertbutyldimethylsilyl)oxy)methyl)hexahydro-1 $H$-pyrrolizin-1-ol (9). Pyrrolidine 7 (81 $\mathrm{mg}, 0.147 \mathrm{mmol}$ ) was dissolved in dry methanol $(2 \mathrm{~mL})$ and cooled to $-78{ }^{\circ} \mathrm{C}$. Ozone was passed through the solution until the reaction was complete. The reaction mixture was quenched with methyl sulfide $(0.1 \mathrm{~mL})$ and allowed to warm to rt. The solvent was evaporated under reduced pressure. The crude aldehyde was immediately employed in the next step without further purification. A solution of the aldehyde in $\mathrm{MeOH}(2 \mathrm{~mL}$ ) was hydrogenated overnight in the presence of $20 \% \operatorname{Pd}(\mathrm{OH})_{2} / \mathrm{C}(0.1 \mathrm{~g})$ at rt. The catalyst was removed by filtration through Celite, and the filtrate was concentrated in vacuo. Purification using silica gel column chromatography gave protected 9 (43 mg, $0.106 \mathrm{mmol})$; yield $72 \%$ for two steps; colorless liquid; $R_{\mathrm{f}}=0.5$ (1 : 2 ethyl acetate-hexanes); $[\alpha]_{\mathrm{D}}^{25}+12.5\left(c 1.0, \mathrm{CHCl}_{3}\right)$; IR (neat) 663, 672, 775, 837, 1016, 1058, 1089, 2855, 2926, $2957 \mathrm{~cm}^{-1}$; ${ }^{1} \mathrm{H} \mathrm{NMR}\left(\mathrm{CDCl}_{3}, 700 \mathrm{MHz}\right) \delta 0.05-0.12(\mathrm{~m}, 12 \mathrm{H}), 0.88-0.95(\mathrm{~m}$, $18 \mathrm{H}), 1.68-1.73(\mathrm{~m}, 2 \mathrm{H}), 1.82-1.84(\mathrm{~m}, 1 \mathrm{H}), 1.95-2.10(\mathrm{~m}, 1 \mathrm{H})$, 2.30-2.40 (br, s, 1H), 2.66-2.74 (m, 1H), 2.80-2.86 (m, 1H), $3.00-3.08(\mathrm{~m}, 1 \mathrm{H}), 3.28-3.32(\mathrm{~m}, 1 \mathrm{H}), 3.60-3.63(\mathrm{~m}, 1 \mathrm{H})$, 3.70-3.92 (m, 2H), $3.91(\mathrm{t}, J=4.8 \mathrm{~Hz}, 1 \mathrm{H}) ;{ }^{13} \mathrm{C} \mathrm{NMR}\left(\mathrm{CDCl}_{3}\right.$, $175 \mathrm{MHz}) \delta-5.2,-5.1,-4.7,-4.1,18.1,18.6,25.4,25.9,26.2$, $30.3,56.3,65.9,70.9,74.0,80.7,82.5$; HRMS (FAB) $m / z:[\mathrm{M}+$ $\mathrm{H}]^{+}$calcd for $\mathrm{C}_{20} \mathrm{H}_{44} \mathrm{O}_{3} \mathrm{NSi}_{2}$ 402.2860, found 402.2861.

$(1 R, 2 R, 3 R, 7 R)-3$-(Hydroxymethyl)hexahydro-1H-pyrrolizine1,2-diol [(+)-hyacinthacine $\left.A_{2}\right]$ (1). The solution of protected 9 $(15.3 \mathrm{mg}, 0.038 \mathrm{mmol})$ in $6 \mathrm{~N} \mathrm{HCl}(2 \mathrm{~mL})$ was refluxed for $12 \mathrm{~h}$. The reaction mixture was then cooled to $\mathrm{rt}$ and evaporated to dryness to give $1 \cdot \mathrm{HCl} ;{ }^{1} \mathrm{H}$ NMR $\left(D_{2} \mathrm{O}, 700 \mathrm{MHz}\right)$ $\delta$ 1.76-1.81 (m, 2H), 1.82-1.90 (m, 1H), 1.91-1.99 (m, 1H), 2.80-2.87 (m, 2H), 2.96-3.01 (m, 1H), 3.27-3.30 (m, 2H), 3.64 (dd, $J=12.0,6.2 \mathrm{~Hz}, 1 \mathrm{H}), 3.73(\mathrm{t}, J=7.9 \mathrm{~Hz}, 1 \mathrm{H}), 3.74-3.80(\mathrm{~m}$, $1 \mathrm{H}) ;{ }^{13} \mathrm{C}$ NMR $\left(\mathrm{D}_{2} \mathrm{O}, 175 \mathrm{MHz}\right) \delta 24.4,29.5,54.9,61.6,66.7$, $69.3,76.5,79.6$. Further purification by treatment of the salt with ion-exchange resin (Dowex $50 \mathrm{~W} \times 8$ ) afforded $1(4.6 \mathrm{mg}$, $0.026 \mathrm{mmol})$; yield 70\%; yellow liquid; $R_{\mathrm{f}}=0.1$ (1:1 methanolchloroform); $[\alpha]_{\mathrm{D}}^{25}+10.4\left(c 0.1, \mathrm{CH}_{3} \mathrm{OH}\right)$ and $+15.3\left(c 0.1, \mathrm{H}_{2} \mathrm{O}\right)$; IR (neat) 1033, 1391, 1642, 2923, $3385 \mathrm{~cm}^{-1} ;{ }^{1} \mathrm{H}$ NMR $\left(\mathrm{D}_{2} \mathrm{O}\right.$, $700 \mathrm{MHz}) \delta 1.85-1.93(\mathrm{~m}, 2 \mathrm{H}), 1.95-2.00(\mathrm{~m}, 1 \mathrm{H}), 2.00-2.06$ $(\mathrm{m}, 1 \mathrm{H}), 2.88-2.96(\mathrm{~m}, 2 \mathrm{H}), 3.05-3.10(\mathrm{~m}, 1 \mathrm{H}), 3.36-3.40(\mathrm{~m}$, $1 \mathrm{H}), 3.71-3.75(\mathrm{~m}, 1 \mathrm{H}), 3.80-3.88(\mathrm{~m}, 3 \mathrm{H}) ;{ }^{13} \mathrm{C}$ NMR $\left(\mathrm{D}_{2} \mathrm{O}\right.$, $175 \mathrm{MHz}) \delta$ 24.4, 29.4, 55.0, 61.2, 66.9, 69.3, 76.3, 79.4; HRMS (FAB) $m / z:[\mathrm{M}+\mathrm{H}]^{+}$calcd for $\mathrm{C}_{8} \mathrm{H}_{16} \mathrm{O}_{3} \mathrm{~N}$ 174.1130, found 174.1128 .

(S)-1-((4R,5R,6R)-5-((tert-Butyldimethylsilyl)oxy)-4-((tertbutyldimethylsilyl)oxy)methyl)-2-phenyl-5,6-dihydro-4H-1,3oxazin-6-yl)allyl acetate (13). Acetic anhydride $(0.863 \mathrm{~mL}$, $9.15 \mathrm{mmol}$ ) and 4-DMAP (224 $\mathrm{mg}, 1.83 \mathrm{mmol}$ ) were added to a stirred solution of alcohol syn-5a (900 mg, $1.83 \mathrm{mmol}$ ) in $\mathrm{CH}_{2} \mathrm{Cl}_{2}(18.3 \mathrm{~mL})$ and pyridine $(1.83 \mathrm{~mL})$. After stirring for $2 \mathrm{~h}$, the reaction mixture was washed with saturated $\mathrm{NH}_{4} \mathrm{Cl}$ solution, saturated $\mathrm{NaHCO}_{3}$ solution, and brine, dried with $\mathrm{MgSO}_{4}$, and evaporated in vacuo. The resulting substance was purified by silica gel column chromatography to give allyl acetate 13 (918 mg, $1.72 \mathrm{mmol}$ ); yield 94\%; colorless oil; $[\alpha]_{\mathrm{D}}^{25}-64.0\left(c\right.$ 1.0, $\mathrm{CHCl}_{3}$ ); IR (neat) 697, 777, 836, 1016, 1033, 1056, 1113, 1233, 1255, 1370, 1470, 1655, 1749, 2859, 2931, 2955, $3732 \mathrm{~cm}^{-1} ;{ }^{1} \mathrm{H} \mathrm{NMR}\left(\mathrm{CDCl}_{3}, 700 \mathrm{MHz}\right) \delta 0.03(\mathrm{~s}, 3 \mathrm{H})$, 0.06-0.07 (m, 3H), 0.10-0.11 (m, 6H), 0.86-0.89 (m, 18H), 2.15 (s, 3H), 3.47 (dd, $J=10.5,8.0 \mathrm{~Hz}, 1 \mathrm{H}), 3.66-3.69(\mathrm{~m}, 1 \mathrm{H}), 3.97$ (dd, $J=10.3,3.9 \mathrm{~Hz}, 1 \mathrm{H}), 4.18(\mathrm{dd}, J=8.2,2.2 \mathrm{~Hz}, 1 \mathrm{H}$ ), 4.23-4.25 (m, 1H), 5.39 (d, $J=10.8 \mathrm{~Hz}, 1 \mathrm{H}), 5.49$ (d, $J=17.2 \mathrm{~Hz}$, 
1H), 5.74-5.78 (m, 1H), 5.91 (ddd, $J=17.1,10.4,6.4 \mathrm{~Hz}, 1 \mathrm{H})$, 7.35-7.37 (m, 2H), 7.40-7.43 (m, 1H), 7.86-7.88 (m, 2H); ${ }^{13} \mathrm{C}$ NMR (175 MHz, $\left.\mathrm{CDCl}_{3}\right) \delta-5.5,-5.4,-4.4,-4.0,18.0,18.1,21.2$, 25.8, 60.6, 63.3, 64.6, 73.1, 75.1, 120.1, 127.2, 128.0, 130.4, 131.7, 133.6, 155.4, 169.8; HRMS (FAB) $m / z:[\mathrm{M}+\mathrm{H}]^{+}$calcd for $\mathrm{C}_{28} \mathrm{H}_{48} \mathrm{O}_{5} \mathrm{NSi}_{2}$ 534.3071, found 534.3069.

(R)-Pentadec-14-en-7-yl acetate (14). 6-Heptenylmagnesium bromide 11 (0.5 M solution in tetrahydrofuran, $5.53 \mathrm{~mL}$, $2.77 \mathrm{mmol}$ ) was added to a dry flask under argon gas. After cooling the mixture to $-40{ }^{\circ} \mathrm{C}$, copper(I) iodide $(0.070 \mathrm{~g}$, $0.37 \mathrm{mmol}$ ) was added to the mixture and stirred for $0.5 \mathrm{~h}$. A solution of $(R)$-epoxyoctane $12(0.23 \mathrm{~g}, 1.84 \mathrm{mmol})$ in THF $(5.1 \mathrm{~mL})$ was added to the mixture and stirred for $3 \mathrm{~h}$. The reaction mixture was treated with MTBE $(50 \mathrm{~mL})$ and saturated $\mathrm{NH}_{4} \mathrm{Cl}$ solution and allowed to warm to rt. The organic layer was separated and the aqueous layer was extracted with ethyl acetate. The combined organic layer was washed with saturated $\mathrm{NaHCO}_{3}$ and brine, dried with $\mathrm{MgSO}_{4}$, and filtered. The filtrate was concentrated in vacuo. Purification using silica gel column chromatography gave unstable secondary alcohol. Acetic anhydride $(0.37 \mathrm{~mL}, 3.88 \mathrm{mmol})$ and 4-DMAP $(0.034 \mathrm{~g}$, $0.277 \mathrm{mmol}$ ) were added to the solution of secondary alcohol in pyridine $(0.32 \mathrm{~mL})$ and stirred for $12 \mathrm{~h}$. The reaction mixture was washed with saturated $\mathrm{NaHCO}_{3}$ and brine, dried with $\mathrm{MgSO}_{4}$ and the filtrate was concentrated in vacuo. The resulting substance was purified by silica gel column chromatography gave acetate $14(0.42 \mathrm{~g}, 1.56 \mathrm{mmol})$; yield 85\% (for 2 steps); colorless oil; $[\alpha]_{\mathrm{D}}^{25}+3.0\left(c\right.$ 1.0, $\mathrm{CHCl}_{3}$ ); IR (neat) 990, 995, 1243, 1373, 1464, 1730, 2858, $2929 \mathrm{~cm}^{-1} ;{ }^{1} \mathrm{H}$ NMR $\left(500 \mathrm{MHz}, \mathrm{CDCl}_{3}\right) \delta$ 0.86-0.89 (m, 3H), 1.26-1.38 (m, 16H), 1.50-1.52 (m, 4H), 2.00-2.10 (m, 5H), 4.80-4.90 (m, 1H), 4.92 $(\mathrm{dd}, J=10.5,2.0 \mathrm{~Hz}, 1 \mathrm{H}), 4.97$ (dd, $J=17.0,2.0 \mathrm{~Hz}, 1 \mathrm{H}) ;{ }^{13} \mathrm{C}$ NMR (125 MHz, $\mathrm{CDCl}_{3}$ ) $\delta 14.2,21.4,22.8,25.4,29.0,29.2,29.4$, 29.6, 31.9, 33.9, 34.3, 74.6, 114.4, 139.3, 171.0; HRMS (FAB) $m / z:[\mathrm{M}+\mathrm{H}]^{+}$calcd for $\mathrm{C}_{17} \mathrm{H}_{33} \mathrm{O}_{2} 269.2481$, found 269.2477 .

$(1 S, 10 R, E)-1-((4 R, 5 R, 6 R)-5-((t e r t-B u t y l d i m e t h y l s i l y l) o x y)-$ 4-((tert-butyldimethylsilyl)oxy)methyl)-2-phenyl-5,6-dihydro-4H1,3-oxazin-6-yl)hexadec-2-ene-1,10-diyl diacetate (15). (R)-Pentadec-14-en-7-yl acetate 14 (1.24 g, $6.14 \mathrm{mmol})$ and Grubbs' second-generation catalyst $(130 \mathrm{mg}, 0.154 \mathrm{mmol})$ were subsequently added to a solution of allyl acetate 13 (1.87 g, $3.51 \mathrm{mmol})$ in $\mathrm{CH}_{2} \mathrm{Cl}_{2}(35 \mathrm{~mL})$ at rt. The reaction mixture was stirred and heated to reflux for $8 \mathrm{~h}$ and then the solvent was removed in vacuo to give the crude products. The resulting substance purified by silica gel column chromatography gave alkene 15 (3.37 g, $4.36 \mathrm{mmol})$; yield 71\%; colorless oil; $[\alpha]_{\mathrm{D}}^{25}$ +31.6 (c 1.0, $\mathrm{CHCl}_{3}$ ); IR (neat) 697, 778, 837, 1022, 1071, 1113, 1137, 1239, 1656, 1749, 2858, 2930, $2954 \mathrm{~cm}^{-1} ;{ }^{1} \mathrm{H}$ NMR $\left(700 \mathrm{MHz}, \mathrm{CDCl}_{3}\right) \delta 0.02-0.04(\mathrm{~m}, 12 \mathrm{H}), 0.76-0.84(\mathrm{~m}, 21 \mathrm{H})$, 1.19-1.22 (m, 14H), 1.31 (br, s, 2H), 1.44 (br, s, 4H), 1.95 (s, $3 \mathrm{H}), 1.97-2.00(\mathrm{~m}, 2 \mathrm{H}), 2.04(\mathrm{~s}, 3 \mathrm{H}), 3.36(\mathrm{t}, J=9.4 \mathrm{~Hz}, 1 \mathrm{H})$, $3.60(\mathrm{br}, \mathrm{s}, 1 \mathrm{H}), 3.91$ (dd, $J=10.4,3.9 \mathrm{~Hz}, 1 \mathrm{H}), 4.07$ (d, $J=$ $8.5 \mathrm{~Hz}, 1 \mathrm{H}), 4.14(\mathrm{~s}, 1 \mathrm{H}), 4.75-4.84(\mathrm{~m}, 1 \mathrm{H}), 5.39(\mathrm{dd}, J=15.4$, $7.7 \mathrm{~Hz}, 1 \mathrm{H}), 5.65(\mathrm{t}, J=8.1 \mathrm{~Hz}, 1 \mathrm{H}), 5.88(\mathrm{dt}, J=15.4,6.0 \mathrm{~Hz}$, $1 \mathrm{H}), 7.19-7.28(\mathrm{~m}, 2 \mathrm{H}), 7.31-7.33(\mathrm{~m}, 1 \mathrm{H}), 7.78(\mathrm{~d}, J=8.4 \mathrm{~Hz}$, $1 \mathrm{H}) ;{ }^{13} \mathrm{C}$ NMR $\left(175 \mathrm{MHz}, \mathrm{CDCl}_{3}\right) \delta-5.5,-5.4,-4.4,-4.0,14.1$,
18.0, 21.3, 22.6, 25.2, 25.3, 25.8, 25.9, 28.7, 29.2, 29.4, 31.8, $32.4,32.5,34.1,60.8,63.4,64.7,73.3,74.3,74.4,74.9,123.2$, $123.3,127.2$, 128.0, 130.4, 133.7, 138.2, 138.4, 155.6, 169.9, 170.9; HRMS (FAB) $m / z$ : $[\mathrm{M}+\mathrm{H}]^{+}$calcd for $\mathrm{C}_{43} \mathrm{H}_{76} \mathrm{O}_{7} \mathrm{NSi}_{2}$ 774.5160 , found 774.5162 .

$(2 R, 3 R, 4 R, 5 S, 14 R, E)-4-($ Benzoyloxy)-2-(((benzyloxy)carbonyl)amino)-3-((tert-butyldimethylsilyl)oxy)-1-hydroxyicos-6-ene5,14-diyl diacetate (16). Benzyl chloroformate $(0.2 \mathrm{~mL}$, $1.40 \mathrm{mmol})$ and a solution of sodium bicarbonate $(0.118 \mathrm{~g}$, $1.40 \mathrm{mmol})$ in $\mathrm{H}_{2} \mathrm{O}(2 \mathrm{~mL})$ were subsequently added to a solution of alkene $15(0.258 \mathrm{~g}, 0.334 \mathrm{mmol})$ in $\mathrm{CH}_{2} \mathrm{Cl}_{2}(2 \mathrm{~mL})$. The mixture was stirred at rt for $24 \mathrm{~h}$. The organic layer was separated and the aqueous layer was extracted with $\mathrm{CH}_{2} \mathrm{Cl}_{2}$. The combined organic layer was washed with saturated $\mathrm{NaHCO}_{3}$ and brine, dried with $\mathrm{MgSO}_{4}$, and filtered. The filtrate was concentrated in vacuo. Purification using silica gel column chromatography (1:15 ethyl acetate-hexanes) gave the carbamate intermediate; colorless oil; $[\alpha]_{\mathrm{D}}^{25}+6.1\left(c\right.$ 1.0, $\left.\mathrm{CHCl}_{3}\right) ;{ }^{1} \mathrm{H}$ NMR (700 MHz, $\left.\mathrm{CDCl}_{3}\right) \delta$ 0.05-0.07 (m, 12H), 0.87-0.88 (m, 21H), 1.20-1.26 (m, 16H), 1.48-1.50 (m, 4H), $1.94(\mathrm{~s}, 5 \mathrm{H}), 2.03$ $(\mathrm{s}, 3 \mathrm{H}), 3.70-3.75(\mathrm{~m}, 1 \mathrm{H}), 3.75-3.80(\mathrm{~m}, 1 \mathrm{H}), 3.90-4.00(\mathrm{~m}$, $1 \mathrm{H}), 4.25(\mathrm{~s}, 1 \mathrm{H}), 4.84(\mathrm{~m}, 1 \mathrm{H}), 5.02-5.10(\mathrm{~m}, 3 \mathrm{H}), 5.42(\mathrm{dd}, J=$ 15.4, 7.2 Hz, 1H), 5.48 (dd, $J=6.5,4.2 \mathrm{~Hz}, 1 \mathrm{H}), 5.76(\mathrm{t}, J=6.9$ $\mathrm{Hz}, 1 \mathrm{H}), 5.87(\mathrm{~m}, 1 \mathrm{H}), 7.26-7.31(\mathrm{~m}, 5 \mathrm{H}), 7.35-7.40(\mathrm{~m}, 2 \mathrm{H})$, 7.50-7.60 (m, 1H), $8.02(\mathrm{~d}, J=8.1 \mathrm{~Hz}, 2 \mathrm{H}) ;{ }^{13} \mathrm{C}$ NMR $(175 \mathrm{MHz}$, $\left.\mathrm{CDCl}_{3}\right) \delta-5.6,-4.6,-4.5,14.0,18.0,18.1,21.0,21.2,22.5,25.2$, $25.7,25.8,28.6,29.0,29.1,31.7,32.3,34.0,54.6,61.2,66.4$, 69.8, 72.8, 74.2, 74.3, 74.6, 124.0, 124.1, 127.8, 128.3, 128.4, 129.6, 129.7, 129.9, 133.0, 136.6, 137.2, 137.3, 155.7, 165.6, 169.9, 170.8. Pyridine $(1.7 \mathrm{~mL})$ buffered HF-pyridine $(0.5 \mathrm{~mL}$, $0.497 \mathrm{mmol})$ was added to a solution of carbamate $(0.24 \mathrm{~g}$, $0.257 \mathrm{mmol})$ in THF $(5 \mathrm{~mL})$ at $0{ }^{\circ} \mathrm{C}$ and then stirred at rt. After $3 \mathrm{~h}$, the reaction mixture was quenched by saturated $\mathrm{NaHCO}_{3}$. The organic layer was separated and the aqueous layer was extracted with ethyl acetate. The combined organic layer was washed with saturated $\mathrm{CuSO}_{4}$ and brine, dried with $\mathrm{MgSO}_{4}$, and the filtrate was concentrated in vacuo. The resulting substance purified by silica gel column chromatography $(1: 15$ ethyl acetate-hexanes) gave primary alcohol 16 (0.173 g, $0.213 \mathrm{mmol}$ ); yield $64 \%$ (for 2 steps); rotamer ratio >20:1; colorless oil; $[\alpha]_{\mathrm{D}}^{25}+8.9\left(c 1.0, \mathrm{CHCl}_{3}\right)$; IR (neat) 713, 778, 838, 1026, 1068, 1108, 1243, 1727, 2857, 2930, $3451 \mathrm{~cm}^{-1} ;{ }^{1} \mathrm{H}$ NMR $\left(700 \mathrm{MHz} \mathrm{CDCl}_{3}\right) \delta-0.06(\mathrm{~s}, 3 \mathrm{H}), 0.00(\mathrm{~s}, 3 \mathrm{H}), 0.75(\mathrm{~s}, 9 \mathrm{H})$, 0.82 (m, 3H), 1.12-1.24 (m, 16H), 1.35-1.50 (m, 4H), 1.85-1.95 (m, 2H), 1.96-2.05 (m, 6H), 3.69 (dd, $J=11.6,3.7 \mathrm{~Hz}, 1 \mathrm{H}), 3.73$ (d, $J=3.7 \mathrm{~Hz}, 1 \mathrm{H}), 3.96$ (d, $J=3.0 \mathrm{~Hz}, 1 \mathrm{H}), 4.23$ (br, s, 1H), 4.73-4.82 (m, 1H), 4.99 (d, $J=12.2 \mathrm{~Hz}, 1 \mathrm{H}), 5.05$ (d, $J=12.1$ $\mathrm{Hz}, 1 \mathrm{H}), 5.30-5.40(\mathrm{~m}, 2 \mathrm{H}), 5.46$ (d, $J=6.9 \mathrm{~Hz}, 1 \mathrm{H}), 5.51-5.59$ $(\mathrm{m}, 1 \mathrm{H}), 5.72-5.80(\mathrm{~m}, 1 \mathrm{H}), 7.25-7.29(\mathrm{~m}, 5 \mathrm{H}), 7.32-7.38(\mathrm{~m}$, 2H), 7.50 (t, $J=7.4 \mathrm{~Hz}, 1 \mathrm{H}), 7.97$ (d, $J=7.2 \mathrm{~Hz}, 2 \mathrm{H}) ;{ }^{13} \mathrm{C} \mathrm{NMR}$ $\left(175 \mathrm{MHz} \mathrm{CDCl}_{3}\right) \delta-4.6,0.0,8.3,14.1,18.0,21.1,21.3,22.6$, 25.2, 25.3, 25.8, 28.6, 29.0, 29.2, 29.3, 31.8, 32.3, 34.1, 53.7, 58.9, 60.4, 62.2, 66.8, 72.2, 72.6, 74.4, 74.8, 123.7, 128.1, 128.5, $129.8,133.3$, 136.4, 137.3, 156.0, 165.8, 170.0, 171.0; HRMS (FAB) $m / z:[\mathrm{M}+\mathrm{H}]^{+}$calcd for $\mathrm{C}_{45} \mathrm{H}_{70} \mathrm{O}_{10} \mathrm{NSi} 812.4769$, found 812.4773 . 


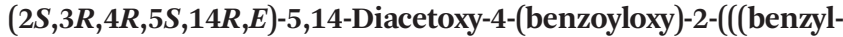
oxy)carbonyl)amino)-3-((tert-butyldimethylsilyl)oxy)icos-6-enoic acid (17). Dess-Martin periodinane (29 $\mathrm{mg}, 0.069 \mathrm{mmol}$ ) was added to a solution of primary alcohol 16 (37.4 mg, $0.046 \mathrm{mmol})$ in $\mathrm{CH}_{2} \mathrm{Cl}_{2}(0.15 \mathrm{~mL})$ at rt. The reaction mixture was stirred at ambient temperature for $2 \mathrm{~h}$. The mixture was diluted with $\mathrm{Et}_{2} \mathrm{O}$, then saturated $\mathrm{NaHCO}_{3}$ (27 mg, $0.322 \mathrm{mmol})$ and $\mathrm{Na}_{2} \mathrm{~S}_{2} \mathrm{O}_{3}(88 \mathrm{mg}, 0.354 \mathrm{mmol})$ were added and then the heterogeneous mixture was stirred at rt. The transparent liquid was washed with brine, dried over $\mathrm{MgSO}_{4}$, and concentrated in vacuo. The resulting substance was immediately used without further purification. 2-Methyl-2butene $(0.33 \mathrm{~mL}), \mathrm{NaH}_{2} \mathrm{PO}_{4}(33.11 \mathrm{mg}, 0.276 \mathrm{mmol})$, and $\mathrm{NaClO}_{2}(24.98 \mathrm{mg}, 0.276 \mathrm{mmol})$ were subsequently added to a solution of the aldehyde in $t$-BuOH $(1.31 \mathrm{ml})$ and $\mathrm{H}_{2} \mathrm{O}$ $(0.33 \mathrm{ml})$ at $\mathrm{rt}$ (2-methyl-2-butene- $t$ - $\left.\mathrm{BuOH}-\mathrm{H}_{2} \mathrm{O}=1: 4: 1\right)$. After $2 \mathrm{~h}$, the mixture was diluted with $\mathrm{H}_{2} \mathrm{O}$ and extracted with ethyl acetate. The organic layer was washed with brine, dried over $\mathrm{MgSO}_{4}$, and the filtrate was concentrated in vacuo. The resulting substance purified by silica gel column chromatography (1:20 methanol-chloroform) gave carboxylic acid 17 (32 $\mathrm{mg}, 0.039 \mathrm{mmol}$ ); yield $84 \%$ (for two steps); rotamer ratio $>20: 1$; colorless oil; $R_{\mathrm{f}}=0.2$ (1:20 methanol-chloroform); $[\alpha]_{\mathrm{D}}^{25}+4.7$ (c 1.0, $\left.\mathrm{CH}_{3} \mathrm{OH}\right)$; IR (neat) 713, 778, 838, 1055, 1067, 1119, 1230, 1248, 1728, 2857, 2930, $3434 \mathrm{~cm}^{-1} ;{ }^{1} \mathrm{H}$ NMR $\left(700 \mathrm{MHz}, \mathrm{CDCl}_{3}\right) \delta-0.16(\mathrm{~s}, 3 \mathrm{H}), 0.00(\mathrm{~s}, 3 \mathrm{H}), 0.67-0.74(\mathrm{~m}$, $9 \mathrm{H}), 0.86-0.88(\mathrm{~m}, 3 \mathrm{H}), 1.14-1.28(\mathrm{~m}, 18 \mathrm{H}), 1.40-1.50(\mathrm{~m}, 4 \mathrm{H})$, 1.90 (br, s, 2H), 2.00-2.10 (m, 3H), $2.12(\mathrm{~s}, 3 \mathrm{H}), 4.41(\mathrm{~s}, 1 \mathrm{H})$, 4.52 (br, s, 1H), 4.80-4.91 (m, 1H), 5.00-5.10 (m, 3H), 5.13 (d, $J=11.6 \mathrm{~Hz}, 1 \mathrm{H}), 5.41$ (br, s, 1H), 5.69-5.78 (m, 4H), 7.25-7.31 (m, 5H), 7.33-7.45 (m, 2H), 7.50-7.60 (m, 1H), 8.02 (d, $J=7.4$ $\mathrm{Hz}, 2 \mathrm{H}) ;{ }^{13} \mathrm{C}$ NMR $\left(175 \mathrm{MHz}, \mathrm{CDCl}_{3}\right) \delta-4.6,-4.5,0.0,14.1$, 18.0, 21.3, 22.6, 25.1, 25.3, 25.7, 28.6, 28.8, 29.2, 29.7, 31.8, $32.1,34.1,56.7,66.8,72.6,72.9,74.8,75.5,124.2,128.1,128.4$, 129.9, 130.2, 133.1, 136.0, 136.5, 155.3, 165.9, 170.8, 171.7; HRMS (FAB) $m / z:[\mathrm{M}+\mathrm{Na}]^{+}$calcd for $\mathrm{C}_{45} \mathrm{H}_{67} \mathrm{O}_{11} \mathrm{NSiNa}$ 848.4381, found 848.4386.

$(2 S, 3 R, 4 R, 5 S, 14 R, E)$-2-Amino-3,4,5,14-tetrahydroxyicos-6-enoic acid [sphingofungin B] (2). $6 \mathrm{~N} \mathrm{HCl}(5 \mathrm{~mL})$ was added to a solution of carboxylic acid 17 (76 $\mathrm{mg}, 0.092 \mathrm{mmol})$ in $\mathrm{MeOH}$ $(5 \mathrm{~mL})$ and then the mixture was stirred at $\mathrm{rt}$ for $7 \mathrm{~h}$ followed by concentration in vacuo. $50 \% \mathrm{KOH}(10 \mathrm{~mL})$ was added to the solution of the residue in $\mathrm{MeOH}(10 \mathrm{~mL})$ and then the reaction mixture was heated at $50{ }^{\circ} \mathrm{C}$ for $24 \mathrm{~h}$. The reaction mixture was cooled to rt, and then Amberlite IRC-76 resin was added to the solution until the $\mathrm{pH}$ value of the solution reached approximately 7.0. The resin was filtered and the filtrate was concentrated in vacuo. Further purification by silica gel column chromatography gave sphingofungin B 2 (14 mg, $0.037 \mathrm{mmol})$; yield $40 \%$ (for two steps); white solid; mp $145-155^{\circ} \mathrm{C}$; $[\alpha]_{\mathrm{D}}^{25}-$ 13.6 (c 1.0, $\mathrm{CH}_{3} \mathrm{OH}$ ); IR (neat) 1014, 1033, 1055, 1409, 1465, 1635, 2855, 2926, $3318 \mathrm{~cm}^{-1} ;{ }^{1} \mathrm{H}$ NMR (700 MHz, CD $\left.{ }_{3} \mathrm{OD}\right)$ $\delta 0.91(\mathrm{t}, J=7.0 \mathrm{~Hz}, 3 \mathrm{H}), 1.32-1.46(\mathrm{~m}, 20 \mathrm{H}), 2.08-2.10(\mathrm{~m}$, 2H), 3.52 (br, s, 1H), $3.64(\mathrm{dd}, J=6.5,1.7 \mathrm{~Hz}, 1 \mathrm{H}), 3.78(\mathrm{~d}, J=$ $4.9 \mathrm{~Hz}, 1 \mathrm{H}), 4.17-4.19$ (m, 2H), 5.51 (dd, $J=15.4,7.4 \mathrm{~Hz}, 1 \mathrm{H})$, $5.80(\mathrm{dt}, J=14.6,6.1 \mathrm{~Hz}, 1 \mathrm{H}) ;{ }^{13} \mathrm{C} \mathrm{NMR}\left(175 \mathrm{MHz}, \mathrm{CD}_{3} \mathrm{OD}\right)$ $\delta 14.5,23.8,26.9,30.4,30.5,30.7,30.8,33.2,33.6,38.6,61.0$, 69.7, 72.6, 75.3, 76.1, 130.4, 135.7, 171.9; HRMS (FAB) $m / z$ : $[\mathrm{M}+\mathrm{H}]^{+}$calcd for $\mathrm{C}_{20} \mathrm{H}_{40} \mathrm{O}_{6} \mathrm{~N} 390.2856$, found 390.2854.

\section{Acknowledgements}

This research was supported by the Basic Science Research Program of the National Research Foundation of Korea (NRF), which is funded by the Ministry of Education, Science and Technology (2010-0022900, 2011-0029199) and by the Yonsung Fine Chemicals Corporation. S.H.P. also gratefully acknowledges the Global Ph. D. Fellowship Grant.

\section{Notes and references}

1 (a) Y. Mu, T. Jin, G.-W. Kim, J.-S. Kim, S.-S. Kim, Y.-S. Tian, C.-Y. Oh and W.-H. Ham, Eur. J. Org. Chem., 2012, 26142620; (b) J.-E. Joo, V.-T. Pham, Y.-S. Tian, Y.-S. Chung, C.-Y. Oh, K.-Y. Lee and W.-H. Ham, Org. Biomol. Chem., 2008, 6, 1498-1501; (c) J.-E. Joo, K.-Y. Lee, V.-T. Pham and W.-H. Ham, Eur. J. Org. Chem., 2007, 1586-1593; (d) J.-E. Joo, K.-Y. Lee, V.-T. Pham, Y.-S. Tian and W.-H. Ham, Org. Lett., 2007, 9, 3627-3630.

2 (a) T. Jin, J.-S. Kim, Y. Mu, S.-H. Park, X. Jin, J.-C. Kang, C.-Y. Oh and W.-H. Ham, Tetrahedron, 2014, 70, 2570-2575; (b) G.-W. Kim, T. Jin, J.-S. Kim, S.-H. Park, K.-H. Lee, S.-S. Kim, I.-S. Myeong and W.-H. Ham, Tetrahedron: Asymmetry, 2014, 25, 87-91; (c) Y. Mu, J.-Y. Kim, X. Jin, S.-H. Park, J.-E. Joo and W.-H. Ham, Synthesis, 2012, 23402346; (d) J.-Y. Kim, Y. Mu, X. Jin, S.-H. Park, V.-T. Pham, D.-K. Song, K.-Y. Lee and W.-H. Ham, Tetrahedron, 2011, 67, 9426-9432; (e) V.-T. Pham, J.-E. Joo, K.-Y. Lee, T.-W. Kim, Y. Mu and W.-H. Ham, Tetrahedron, 2010, 66, 2123-2131.

3 For recent reviews, see: (a) P. Compain and O. R. Martin, Iminosugar: From Synthesis to Therapeutic Applications, Wiley, Chichester, 2007; (b) W. Zou, Curr. Top. Med. Chem., 2005, 5, 1363-1391; (c) Y. Nishimura, Curr. Top. Med. Chem., 2003, 3, 575-591; (d) P. Compain and O. R. Martin, Curr. Top. Med. Chem., 2003, 3, 541-560; (e) T. M. Wrodnigg, Monatsh. Chem., 2002, 133, 393-426; $(f)$ A. A. Watson, G. W. J. Fleet, N. Asano, R. J. Molyneux and R. J. Nash, Phytochemistry, 2001, 56, 265-295; (g) A. E. Stütz, Iminosugars as Glycosidase Inhibitors: Nojirimycin and Beyond, Wiley-VCH, Weinheim, 1999.

4 N. Asano, H. Kuroi, K. Ikeda, H. Kizu, Y. Kameda, A. Kato, I. Adachi, A. A. Watson, R. J. Nash and G. W. J. Fleet, Tetrahedron: Asymmetry, 2000, 11, 1-8.

5 For previous syntheses of (+)-hyacinthacine $\mathrm{A}_{2}$, see: (a) M. Royzen, M. T. Taylor, A. DeAngelis and J. M. Fox, Chem. Sci., 2011, 2, 2162-2165; (b) X.-K. Liu, S. Qiu, Y.-G. Xiang, Y.-P. Ruan, X. Zheng and P.-Q. Huang, J. Org. Chem., 2011, 76, 4952-4963; (c) C. Bonaccini, M. Chioccioli, C. Parmeggiani, F. Cardona, D. Lo Re, G. Soldaini, P. Vogel, 
C. Bello, A. Goti and P. Gratteri, Eur. J. Org. Chem., 2010, 5574-5585; (d) W.-J. Liu, J.-L. Ye and P.-Q. Huang, Org. Biomol. Chem., 2010, 8, 2085-2091; (e) I. Delso, T. Tejero, A. Goti and P. Merino, Tetrahedron, 2010, 66, 1220-1227; (f) C. Ribes, E. Falomir, M. Carda and J. A. Marco, Tetrahedron, 2009, 65, 6965-6971; $(g)$ P. Dewi-Wülfing and S. Blechert, Eur. J. Org. Chem., 2006, 1852-1856; (h) S. Desvergnes, S. Py and Y. Vallée, J. Org. Chem., 2005, 70, 1459-1462; (i) I. Izquierdo, M. T. Plaza and F. Franco, Tetrahedron: Asymmetry, 2003, 14, 3933-3935; (j) F. Cardona, E. Faggi, F. Liguori, M. Cacciarini and A. Goti, Tetrahedron Lett., 2003, 44, 2315-2318; (k) L. Rambaud, P. Compain and O. R. Martin, Tetrahedron: Asymmetry, 2001, 12, 1807-1809.

6 For previous syntheses of (-)-hyacinthacine $\mathrm{A}_{2}$, see: (a) E. A. Brock, S. G. Davies, J. A. Lee, P. M. Roberts and J. E. Thomson, Org. Biomol. Chem., 2013, 11, 3187-3202; (b) J. Calveras, J. Casas, T. Parella, J. Joglar and P. Clapés, Adv. Synth. Catal., 2007, 349, 1661-1666.

7 For recent reviews, see: (a) H.-S. Byun, X. Lu and R. Bittman, Synthesis, 2006, 2447-2474; (b) J. Liao, J. Tao, G. Lin and D. Liu, Tetrahedron, 2005, 61, 4715-4733; (c) S. H. Kang, S. Y. Kang, H.-S. Lee and A. J. Buglass, Chem. Rev., 2005, 105, 4537-4558; (d) Y. Ohfune and T. Shinada, Eur. J. Org. Chem., 2005, 5127-5143; (e) M. Brunner and A. M. P. Koskinen, Curr. Org. Chem., 2004, 8, 1629-1645.

8 (a) F. VanMiddlesworth, R. A. Giacobbe, M. Lopez, G. Garrity, J. A. Bland, K. Bartizal, R. A. Fromtling, J. Polishook, M. Zweerink, A. M. Edison, W. Rozdilsky, K. E. Wilson and R. L. Monaghan, J. Antibiot., 1992, 45, 861; (b) F. VanMiddlesworth, C. Dufresne, F. E. Wincott, R. T. Mosley and K. E. Wilson, Tetrahedron Lett., 1992, 33, 297-300.

9 For previous syntheses of sphingofungin B, see: (a) S. Kobayashi and T. Furuta, Tetrahedron, 1998, 54, 10275-10294; (b) S. Kobayashi, T. Furuta, T. Hayashi, M. Nishijima and K. Hanada, J. Am. Chem. Soc., 1998, 120, 908-919; (c) S. Kobayashi, T. Hayashi, S. Iwamoto, T. Furuta and M. Matsumura, Synlett, 1996, 672-674.

10 For previous syntheses of sphingofungin D, see: (a) K. Otaka and K. Mori, Eur. J. Org. Chem., 1999, 17951802; (b) N. Chida, H. Ikemoto, A. Noguchi, S. Amano and S. Ogawa, Nat. Prod. Lett., 1995, 6, 295-302; (c) K. Mori and K. Otaka, Tetrahedron Lett., 1994, 35, 9207-9210.

11 For previous syntheses of sphingofungin E, see: (a) K. Ikeuchi, M. Hayashi, T. Yamamoto, M. Inai, T. Asakawa, Y. Hamashima and T. Kan, Eur. J. Org. Chem., 2013, 6789-6792; (b) M. Martinková, J. Gonda, J. Š. Raschmanová, M. Slaninková and J. Kuchár, Carbohydr. Res., 2010, 345, 2427-2437; (c) B. Wang and G.-Q. Lin, Eur. J. Org. Chem., 2009, 5038-5046; (d) C. J. Hayes, D. M. Bradley and N. M. Thomson, J. Org. Chem., 2006, 71, 2661-2665; (e) T. Oishi, K. Ando, K. Inomiya, H. Sato, M. Iida and N. Chida, Bull. Chem. Soc. Jpn., 2002, 75, 19271947; (f) T. Oishi, K. Ando, K. Inomiya, H. Sato, M. Iida and N. Chida, Org. Lett., 2002, 4, 151-154; $(g)$ T. Nakamura and M. Shiozaki, Tetrahedron, 2002, 58, 8779-8791; (h) B. M. Trost and C. Lee, J. Am. Chem. Soc., 2001, 123, 12191-12201; (i) T. Nakamura and M. Shiozaki, Tetrahedron Lett., 2001, 42, 2701-2704; $(j)$ B. Wang, X.-M. Yu and G.-Q. Lin, Synlett, 2001, 904-906.

12 For previous syntheses of sphingofungin $F$, see: (a) Y.-C. Luo, H.-H. Zhang, Y. Wang and P.-F. Xu, Acc. Chem. Res., 2010, 43, 1317-1330; (b) F.-F. Gan, S.-B. Yang, Y.-C. Luo, W.-B. Yang and P.-F. Xu, J. Org. Chem., 2010, 75, 2737-2740; (c) See ref. 11c; (d) M. Li and A. Wu, Synlett, 2006, 2985-2988; (e) K.-Y. Lee, C.-Y. Oh and W.-H. Ham, Org. Lett., 2002, 4, 4403-4405; $(f)$ See ref. 11h; $(g)$ D.-G. Liu, B. Wang and G.-Q. Lin, J. Org. Chem., 2000, 65, 9114-9119; (h) See ref. $9 a$; (i) B. M. Trost and C. B. Lee, J. Am. Chem. Soc., 1998, 120, 6818-6819; (j) S. Kobayashi, M. Matsumura, T. Furuta, T. Hayashi and S. Iwamoto, Synlett, 1997, 301-303.

13 For previous syntheses of myriocin, see: (a) M. Inai, T. Goto, T. Furuta, T. Wakimoto and T. Kan, Tetrahedron: Asymmetry, 2008, 19, 2771-2773; (b) M. C. Jones and S. P. Marsden, Org. Lett., 2008, 10, 4125-4128; (c) K.-Y. Lee, C.-Y. Oh, Y.-H. Kim, J.-E. Joo and W.-H. Ham, Tetrahedron Lett., 2002, 43, 9361-9363 and references cited therein; (d) See ref. 11e; (e) T. Oishi, K. Ando and N. Chida, Chem. Commun., 2001, 1932-1933.

14 For previous syntheses of mycestericin A, see: (a) H. Yamanaka, K. Sato, H. Sato, M. Iida, T. Oishi and N. Chida, Tetrahedron, 2009, 65, 9188-9201; (b) H. Sato, K. Sato, M. Iida, H. Yamanaka, T. Oishi and N. Chida, Tetrahedron Lett., 2008, 49, 1943-1947.

15 For previous synthesis of mycestericin C, see: S. Sakamoto, N. Kazumi, Y. Kobayashi, C. Tsukano and Y. Takemoto, Org. Lett., 2014, 16, 4758-4761.

16 For reviews, see: (a) A. Mengel and O. Reiser, Chem. Rev., 1999, 99, 1191-1223; (b) M. T. Reetz, Chem. Rev., 1999, 99, 1121-1162; (c) M. T. Reetz, Angew. Chem., Int. Ed. Engl., 1991, 30, 1531-1546; (d) J. Jurczak and A. Gołębiowski, Chem. Rev., 1989, 89, 149-164; (e) M. T. Reetz, Angew. Chem., Int. Ed. Engl., 1984, 23, 556-569.

17 R. S. Coleman and A. J. Carpenter, Tetrahedron Lett., 1992, 33, 1697-1700.

18 T. Fujisawa, Y. Ukaji, M. Funabora, M. Yamashita and T. Sato, Bull. Chem. Soc. Jpn., 1990, 63, 1894-1897.

19 G. Boireau, A. Deberly and D. Abenhaïm, Tetrahedron, 1989, 45, 5837-5844.

20 S. Kobayashi, P. Das, G. X. Wang, T. Mita, M. J. Lear and M. Hirama, Chem. Lett., 2002, 300-301.

21 (a) M. Asami and R. Kimura, Chem. Lett., 1985, 1221-1222; (b) M. Asami and T. Mukaiyama, Chem. Lett., 1983, 93-96.

22 R. Bloch and L. Gilbert, Tetrahedron Lett., 1987, 28, 423-426.

23 G. E. Keck, M. B. Andrus and D. R. Romer, J. Org. Chem., 1991, 56, 417-420.

24 J. Mulzer and C. Pietschmann, J. Org. Chem., 1997, 62, 3938-3943.

25 "Although the actual role of $\mathrm{ZnBr}_{2}$ is not clear at present" by Asami (ref. 21a, p. 1222), "its generality is unknown" by 
Reetz (ref. 16e, p. 562), "although the actual stereochemical course is still an open question" by Fujisawa (ref. 18, p. 1895), "argues further against simple chelation model" by Coleman (ref. 17, p. 1698), and "the actual role of $\mathrm{Ti}$ $(\mathrm{OiPr})_{4}$ in our case... in THF is still a matter of debate" by Hirama (ref. 20, p. 301).
26 (a) J. L. von dem Bussche-Hünnefeld and D. Seebach, Tetrahedron, 1992, 48, 5719-5730; (b) D. Seebach, L. Behrendt and D. Felix, Angew. Chem. Int., Ed. Engl., 1991, 30, 10081009.

27 I. Izquierdo, M. T. Plaza, R. Robles and F. Franco, Tetrahedron: Asymmetry, 2001, 12, 2481-2487. 